\title{
ON TIME OPTIMAL CONTROL OF THE WAVE EQUATION AND ITS NUMERICAL REALIZATION AS PARAMETRIC OPTIMIZATION PROBLEM*
}

\author{
$\mathrm{KARL} \mathrm{KUNISCH}^{\dagger}$ AND DANIEL WACHSMUTH ${ }^{\ddagger}$
}

\begin{abstract}
Time optimal control of the wave equation is analyzed on the basis of a regularized formulation which is considered as a bilevel optimization problem. For the lower level problems, which are constrained optimal control problems for the wave equation, a detailed sensitivity analysis is carried out. Further a semismooth Newton method is analyzed and proved to converge locally superlinearly. Numerical examples are provided.
\end{abstract}

Key words. time optimal control, wave equation, optimality condition, sensitivity analysis, semismooth Newton method

AMS subject classifications. 49M15, 49K20

DOI. $10.1137 / 120877520$

1. Introduction. This paper is devoted to the time optimal control problem

$$
\left\{\begin{array}{l}
\min \int_{0}^{\tau} d t \\
\text { subject to } \tau \geq 0, \\
y_{t t}-\Delta y=\chi_{\omega} u \text { in }(0, \tau) \times \Omega, \\
y(0)=y_{1}, y_{t}(0)=y_{2}, y(\tau)=z_{1}, y_{t}(\tau)=z_{2} \text { in } \Omega, \\
y=0 \text { on } \Gamma, \\
\|u(t)\|_{L^{2}(\omega)} \leq \gamma \text { for a.e. } t \in(0, \tau) .
\end{array}\right.
$$

Here, $\gamma>0$ is a fixed positive constant and $\Omega \subset \mathbb{R}^{n}, n \geq 1$ is a fixed bounded domain with a $C^{2}$ boundary $\Gamma$. Further $\omega \subset \Omega$ is a measurable subset and $\chi_{\omega} u$ denotes the extension-by-zero operator from $\omega$ to $\Omega$. The initial and terminal states are fixed and - unless otherwise specified — are assumed to satisfy

$$
y_{1} \in H_{0}^{1}(\Omega), z_{1} \in H_{0}^{1}(\Omega), y_{2} \in L^{2}(\Omega), z_{2} \in L^{2}(\Omega) .
$$

In previous work [19] we used a regularization scheme to derive an optimality system for $(\tilde{P})$ and in particular to investigate the maximum principle and the transversality condition for $(\tilde{P})$. The present work aims at solving $(\tilde{P})$ on the basis of this regularization scheme.

Let us briefly explain the route that we will follow. The regularized time optimal control problem is considered as a bilevel optimization problem. The lower level con-

*Received by the editors May 16, 2012; accepted for publication (in revised form) January 7, 2013; published electronically March 21, 2013.

http://www.siam.org/journals/sicon/51-2/87752.html

$\dagger$ Institute for Mathematics and Scientific Computing, A-8010 Graz, Austria (karl.kunisch@ unigraz.at, http://www.kfunigraz.ac.at/imawww/kunisch). This author was partially supported by "Fonds zur Förderung der Wissenschaftlichen Forschung" under SFB 32, Mathematical Optimization and Applications in the Biomedical Sciences.

${ }^{\ddagger}$ Institute of Mathematics, University of Würzburg, D-97074 Würzburg, Germany, and Johann Radon Institute for Computational and Applied Mathematics (RICAM), Austrian Academy of Sciences, A-4040 Linz, Austria (daniel.wachsmuth@mathematik.uni-wuerzburg.de). 
sists of constrained optimal control problems for the wave equation, and the upper level problems are minimization problems with respect to $\tau$. Alternatively we can consider our approach as a reduced approach, with the optimal time the only independent optimization variable, and the control a dependent variable. The lower level problems are parameterized optimization problems in the parameter $\tau$. We establish Bouligand differentiability of the solutions to these lower level problems. This allows us to obtain second-order directional differentiability of the cost of the parameterized optimization problems. Moreover we show that setting the first derivative of the cost equal to zero corresponds to the transversality condition in the optimality system. This structural result is not restricted to the case where the constrained equation is the wave equation. The solution of the lower level problem requires efficient solution techniques. For this purpose we analyze a semismooth Newton method. This necessitates that we establish Newton differentiability of the projection onto the unit ball in $L^{2}$, a result which is of interest in its own right. Finally there is an outermost iteration within which the regularization parameter is driven to zero.

The development of numerical techniques for time optimal control problems has received much attention in the context of ordinary differential equations. They are frequently categorized into direct and indirect methods. Indirect methods based on multiple shooting techniques $[2,14]$ solve the two-point boundary value problem describing first-order necessary conditions. In [12] a semismooth Newton method was recently proposed for solving the nonsmooth optimality systems. Direct methods, on the other hand, consider time optimal problems as genuine nonlinear programming problems. They are used in several variants, which frequently involve reparametrization of the controls as the unknowns. The new unknowns can be the switching times as in [22] or the arc durations as in [13].

Time optimal control for the wave equation was frequently investigated in previous work, mostly with the aim of deriving different forms of the maximum principle. We refer, e.g., to Fattorini [4, 5], Gugat and Leugering [8], and Krabs [16, 17] and the references given there. In this context it was also discussed that time optimal control problems can be addressed alternatively by solving appropriately defined dual norm optimal control problems, which are parameterized by the time $\tau$; see, e.g., $[6,16]$. If for some parameter value $\hat{\tau}$ the norm optimal control satisfies $\|\hat{u}\|_{L^{\infty}\left(I ; L^{2}(\omega)\right)}=\gamma$, then $(\hat{\tau}, \hat{u})$ is a solution of $(\tilde{P})$. However, this equivalence is typically established only for the special case $\omega=\Omega$. An example in [8] shows that the equivalence of time optimal and norm optimal control problems cannot be expected in the general case. In [9] the relationship between norm- and time optimal problems was utilized to develop a method-of-moments-based algorithm to solve time optimal control problems for onedimensional vibrating systems. In a recent paper Wang and Zuazua [25] analyzed the equivalence of time and norm optimal control problems for the heat equation for the case $\omega \neq \Omega$. The proof of equivalence uses two properties that are not available for the wave equation: unconstrained null-controllability for arbitrarily small times and the bang-bang property of time optimal controls. Hence addressing the time optimal control problem for the wave equation in the general situation $\omega \neq \Omega$ by means of the dual norm optimal control problem remains to be an independent challenge.

The paper is organized as follows. In section 2 we introduce the abstract form of the wave equation and of problem $(\tilde{P})$ and recall selected results from [19]. Section 3 is devoted to the sensitivity analysis of a parametric family of optimization problems parameterized in the variable $\tau$. In section 4 we give the analysis of the semismooth Newton method for the lower level problems. Section 5 contains a description of the numerical algorithm and selected numerical experiments. 
Function spaces. Let us briefly recall definitions of function spaces and their scalar products and norms, which will be used throughout the paper. We will frequently use the standard spaces $L^{2}(\Omega), H_{0}^{1}(\Omega)$ endowed with their usual scalar product

$$
(u, v)_{L^{2}(\Omega)}=\int_{\Omega} u(x) v(x) d x, \quad(u, v)_{H_{0}^{1}(\Omega)}=\int_{\Omega} \nabla u(x) \cdot \nabla v(x) d x .
$$

Moreover, we will work with spaces of abstract functions $C(\bar{I} ; X)$ and $L^{2}(I ; X)$, where $X$ is a Hilbert space and $I=(0,1)$. The scalar product in $L^{2}(I ; X)$ and the norm in $C(\bar{I} ; X)$ is defined by

$$
(u, v)_{L^{2}(X)}=\int_{0}^{1}(u(t), v(t))_{X} d t, \quad\|u\|_{C(\bar{I} ; X)}=\max _{t \in[0,1]}\|u(t)\|_{X} .
$$

\section{Preliminaries.}

2.1. Abstract formulation. Let us recall some facts concerning the wave equation

$$
\begin{cases}y_{t t}-\Delta y=\chi_{\omega} u & \text { in }(0, \tau) \times \Omega, \\ y(0)=y_{1}, y_{t}(0)=y_{2} & \text { in } \Omega, \\ y=0 & \text { on } \Gamma\end{cases}
$$

with $\tau>0$ fixed that will be relevant to our work. For the purpose of numerical realization it is convenient to perform a transformation of (2.1) to the fixed time interval

$$
I:=(0,1)
$$

Moreover, to express (2.1) in abstract form we introduce the operators

$$
\mathbf{A}:=\left(\begin{array}{cc}
0 & I \\
\Delta & 0
\end{array}\right), \quad \mathbf{B}:=\left(\begin{array}{c}
0 \\
\chi_{\omega}
\end{array}\right)
$$

and vectors

$$
\mathbf{y}_{0}:=\left(\begin{array}{l}
y_{1} \\
y_{2}
\end{array}\right), \mathbf{z}:=\left(\begin{array}{c}
z_{1} \\
z_{2}
\end{array}\right), \mathbf{y}(t):=\left(\begin{array}{l}
\mathbf{y}_{1}(t) \\
\mathbf{y}_{2}(t)
\end{array}\right)
$$

Then the wave equation (2.1) can be expressed as the first-order evolution equation

$$
\begin{aligned}
\mathbf{y}_{t} & =\tau(\mathbf{A y}+\mathbf{B} u) \quad \text { on }(0,1], \\
\mathbf{y}(0) & =\mathbf{y}_{0} .
\end{aligned}
$$

The components of the solution $\mathbf{y}$ of this equation fulfill $\left(\mathbf{y}_{1}\right)_{t}=\tau \mathbf{y}_{2}$ and $\left(\mathbf{y}_{2}\right)_{t}=$ $\tau\left(\Delta \mathbf{y}_{1}+\chi_{\omega} u\right)$. For convenience of notation we introduce the function spaces

$$
Y^{s}= \begin{cases}L^{2}(\Omega), & s=0, \\ H^{s}(\Omega) \cap H_{0}^{1}(\Omega), & s=1,2, \\ H_{0}^{1}(\Omega)^{*}, & s=-1\end{cases}
$$

and the associated vector-valued spaces, which take account of the regularity of the components of solutions $\mathbf{y}$ of (2.2):

$$
\mathbf{Y}^{s}:=Y^{s} \times Y^{s-1}
$$

Copyright $@$ by SIAM. Unauthorized reproduction of this article is prohibited. 
By assumption we have $\mathbf{y}_{0} \in \mathbf{Y}^{1}$. Utilizing this notation the operator $\mathbf{A}$ is a continuous linear operator in the following sense:

$$
\mathbf{A} \in \mathcal{L}\left(\mathbf{Y}^{s}, \mathbf{Y}^{s-1}\right) .
$$

Moreover, the operator $\mathbf{B}$ has the property

$$
\mathbf{B} \in \mathcal{L}\left(L^{2}(\omega), \mathbf{Y}^{1}\right)
$$

Next we transform $(\tilde{P})$ to the interval $I$ and express it in abstract form. For this purpose we introduce the set of admissible controls

$$
U_{a d}:=\left\{u \in L^{\infty}\left(I ; L^{2}(\omega)\right): u(t) \in U \text { a.e. on } I\right\}
$$

with $U$ given by

$$
U=\left\{u \in L^{2}(\omega):\|u\|_{L^{2}(\omega)} \leq \gamma\right\} .
$$

Now $(\tilde{P})$ can be expressed as

$$
\left\{\begin{array}{l}
\min \tau \\
\text { subject to } \tau \geq 0 \text { and } \\
\mathbf{y}_{t}=\tau(\mathbf{A y}+\mathbf{B} u) \text { on }(0,1] \\
\mathbf{y}(0)=\mathbf{y}_{0}, \mathbf{y}(1)=\mathbf{z}, \\
u \in U_{a d}
\end{array}\right.
$$

For existence and uniqueness of weak solutions of system (2.2), we have the following well-known result; see, e.g., [3, 20].

Theorem 2.1. Let $\mathbf{y}_{0} \in \mathbf{Y}^{1}, u \in L^{2}\left(I ; L^{2}(\omega)\right)$ be given. Then the first-order equation (2.2) admits a unique weak solution $\mathbf{y}$ that satisfies

$$
\mathbf{y} \in C\left(\bar{I} ; \mathbf{Y}^{1}\right), \mathbf{y}_{t} \in L^{2}\left(I ; \mathbf{Y}^{0}\right) .
$$

If, moreover, $\mathbf{y}_{0} \in \mathbf{Y}^{2}, u_{t} \in L^{2}\left(I ; L^{2}(\omega)\right)$, then

$$
\mathbf{y} \in C\left(\bar{I} ; \mathbf{Y}^{2}\right), \mathbf{y}_{t} \in C\left(\bar{I} ; \mathbf{Y}^{1}\right) .
$$

In either of these cases the solution $\mathbf{y}$ depends continuously on $\left(u, \mathbf{y}_{0}\right)$ in the indicated norms.

Together with the primal equation (2.2), the adjoint equation will play a central role. It is defined as the evolution equation

$$
-\mathbf{p}_{t}=\tau \mathbf{A}^{*} \mathbf{p} \quad \text { on } I .
$$

Here $\mathbf{A}^{*}$ is given as adjoint of $\mathbf{A}$ :

$$
\mathbf{A}^{*}:=\left(\begin{array}{cc}
0 & \Delta \\
I & 0
\end{array}\right)
$$

Hence, (2.3) is a wave equation in the second coordinate $\mathbf{p}_{2}$ with $\left(\mathbf{p}_{2}\right)_{t}=-\tau \mathbf{p}_{1}$ and

$$
\left(\mathbf{p}_{2}\right)_{t t}=\tau^{2} \Delta \mathbf{p}_{2}
$$

Copyright $@$ by SIAM. Unauthorized reproduction of this article is prohibited. 
It will be convenient to introduce the notation

$$
\mathbf{P}^{s}:=Y^{s-1} \times Y^{s},
$$

which will be used for $s=0,1,2$. The index $s$ with $\mathbf{Y}^{s}$ and $\mathbf{P}^{s}$ denotes the regularity of the wave function for the primal state $\mathbf{y}$ and the adjoint state $\mathbf{p}$, respectively. We may note that $\left(\mathbf{Y}^{s}\right)^{*}=\mathbf{P}^{(1-s)}$.

If the adjoint equation is completed with a terminal condition $\mathbf{p}(1)=\hat{\mathbf{p}}$ with $\hat{\mathbf{p}} \in \mathbf{P}^{0}$, then it is uniquely solvable with solution $\mathbf{p} \in C\left(\bar{I} ; \mathbf{P}^{0}\right)$. Moreover, one has regularity results analogous to those for the primal wave equation expressed in Theorem 2.1.

To guarantee well-posedness of $(P)$ we need to assume that the set of feasible controls is nonempty. For this purpose we assume that

$$
\text { the wave equation (2.2) is null-controllable, }
$$

i.e., there exists some $\tau_{0}>0$ and $c>0$ such that

$$
\|\mathbf{p}(1)\|_{\mathbf{P}^{0}}^{2} \leq c\left\|\mathbf{B}^{*} \mathbf{p}\right\|_{L^{2}\left(I ; L^{2}(\omega)\right)}^{2}
$$

for every solution to the adjoint equation (2.3) with $\tau=\tau_{0}$. Based on the Hilbert uniqueness method, cf. [21], it is well established that the exact controllability problem for the wave equation can be reduced to the analysis of inequality (2.4), see, e.g., [26], for a survey on techniques that were developed to analyze controllability for the wave equation. In particular null-controllability of the wave equation with distributed (or internal) controls holds if the geometric control condition is satisfied: every ray of geometric optics propagating in the domain $\Omega$ hits the subset $\omega$ within time less than $\tau_{0}$; see [1]. The method of moments and fundamental solutions were used in [24] to establish controllability. In [10], on the basis of spectral techniques, sufficient conditions for exact controllability are derived for the case of controls that are less regular than $L^{2}$.

With (H1) holding it can be shown that there exists a feasible solution to $(P)$ for $\tau$ large enough $[19,23]$, and then existence to $(P)$ readily follows.

THEOREM 2.2. Let $\mathbf{y}_{0}, \mathbf{z} \in \mathbf{Y}^{1}$ be given. If there is $\tau_{0}>0$ such that the waveequation is null-controllable, i.e., (2.4) holds, then the time optimal control problem $(\tilde{P})$ admits a solution.

Proof. We refer to [19, Thm. 2.4].

In what follows, we will denote the uniquely determined minimal time by $\tau^{*}$.

2.2. A family of regularized problems. To derive an optimality system for $(P)$, the following family of problems was considered in [19], which involves a regularization of the control and a penalization of the terminal constraint: For $\varepsilon>0$ we consider

$\left(P_{\varepsilon}\right)$

$$
\left\{\begin{array}{l}
\min J_{\varepsilon}(\tau, u)=\tau\left(1+\frac{\varepsilon}{2}\|u\|_{L^{2}\left(I ; L^{2}(\omega)\right)}^{2}\right)+\frac{1}{2 \varepsilon}\|\mathbf{y}(1)-\mathbf{z}\|_{\mathbf{Y}^{0}}^{2} \\
\text { subject to } \tau \geq 0 \text { and } \\
\mathbf{y}_{t}=\tau \mathbf{A y}+\tau \mathbf{B} u, \text { on }(0,1], \\
\mathbf{y}(0)=\mathbf{y}_{0}, \\
u \in U_{a d} .
\end{array}\right.
$$


Here and below the norm on $\mathbf{Y}^{0}=L^{2}(\Omega) \times H^{-1}(\Omega)$ is chosen to be

$$
\|\mathbf{v}\|_{\mathbf{Y}^{0}}^{2}=\left\|v_{1}\right\|_{L^{2}(\Omega)}^{2}+\left((-\Delta)^{-1} v_{2}, v_{2}\right)_{L^{2}(\Omega)},
$$

where $w=(-\Delta)^{-1} v_{2}$ is the solution of $-\Delta w=v_{2}$ in $\Omega, w=0$ on $\Gamma$.

For any $\varepsilon>0$ problem $\left(P_{\varepsilon}\right)$ admits a solution $\left\{\left(\tau_{\varepsilon}, \mathbf{y}_{\varepsilon}, u_{\varepsilon}\right)\right\}$ independently of (H1). In order to guarantee convergence of these solutions to a solution of $(P)$, it is, of course, required to assume (H1).

TheOrEm 2.3. Assume that (H1) holds and let $\left\{\left(\tau_{\varepsilon}, \mathbf{y}_{\varepsilon}, u_{\varepsilon}\right)\right\}_{\varepsilon>0}$ denote a family of solutions of $\left(P_{\varepsilon}\right)$. Then we have that

$$
\tau_{\varepsilon} \rightarrow \tau^{*} \text { for } \varepsilon \rightarrow 0^{+}
$$

and $\left(\mathbf{y}_{\varepsilon}, u_{\varepsilon}\right)$ is uniformly bounded in $\left(C\left(\bar{I} ; \mathbf{Y}^{1}\right) \cap H^{1}\left(I ; \mathbf{Y}^{0}\right)\right) \times L^{\infty}\left(T ; L^{2}(\omega)\right)$. Moreover, for each weakly star converging subsequence $\left\{\left(\mathbf{y}_{\varepsilon_{n}}, u_{\varepsilon_{n}}\right)\right\}$ with

$$
\mathbf{y}_{\varepsilon_{n}} \rightarrow^{*} \tilde{\mathbf{y}} \text { in } L^{\infty}\left(I ; \mathbf{Y}^{1}\right) \cap H^{1}\left(I ; \mathbf{Y}^{0}\right), \quad u_{\varepsilon_{n}} \rightarrow^{*} \tilde{u} \text { in } L^{\infty}\left(I ; L^{2}(\omega)\right),
$$

the limit $\left(\tau^{*}, \tilde{\mathbf{y}}, \tilde{u}\right)$ is a solution of the original time optimal control problem $(P)$.

If $\tilde{u}$ is bang-bang, then the convergence $\left(\mathbf{y}_{\varepsilon_{n}}, u_{\varepsilon_{n}}\right) \rightarrow(\tilde{\mathbf{y}}, \tilde{u})$ is strong in $\left(C\left(\bar{I} ; \mathbf{Y}^{1}\right) \cap\right.$ $\left.H^{1}\left(I ; \mathbf{Y}^{0}\right)\right) \times L^{2}\left(I ; L^{2}(\omega)\right)$.

This result was proved in [19]. There $\left(P_{\varepsilon}\right)$ was also used to investigate the firstorder optimality condition for $(P)$, involving the maximum principle and the transversality condition. In the present work we focus on the numerical realization of $\left(P_{\varepsilon}\right)$. It is based on the necessary optimality conditions for $\left(P_{\varepsilon}\right)$ from [19], which we recall next.

TheOrem 2.4. Let $\left(\tau_{\varepsilon}, \mathbf{y}_{\varepsilon}, u_{\varepsilon}\right)$ be a local solution of $\left(P_{\varepsilon}\right)$. Then there exists $\mathbf{p}_{\varepsilon} \in C\left(\bar{I} ; \mathbf{P}^{2}\right) \cap C^{1}\left(\bar{I} ; \mathbf{P}^{1}\right)$ such that the following optimality system holds:

$$
\left\{\begin{array}{l}
\partial_{t} \mathbf{y}_{\varepsilon}=\tau_{\varepsilon} \mathbf{A y}_{\varepsilon}+\tau_{\varepsilon} \mathbf{B} u_{\varepsilon}, \quad \mathbf{y}_{\varepsilon}(0)=\mathbf{y}_{0}, \\
-\partial_{t} \mathbf{p}_{\varepsilon}=\tau_{\varepsilon} \mathbf{A}^{*} \mathbf{p}_{\varepsilon}, \quad \mathbf{p}_{\varepsilon}(1)=\frac{1}{\varepsilon}\left(\begin{array}{c}
\mathbf{y}_{\varepsilon, 1}(1)-z_{1} \\
(-\Delta)^{-1}\left(\mathbf{y}_{\varepsilon, 2}(1)-z_{2}\right)
\end{array}\right) \in \mathbf{P}^{2}, \\
\left(\varepsilon u_{\varepsilon}+\mathbf{B}^{*} \mathbf{p}_{\varepsilon}, u-u_{\varepsilon}\right)_{L^{2}\left(I ; L^{2}(\omega)\right) \geq 0 \text { for all } u \in U_{a d},} \\
1+\frac{\varepsilon}{2}\left\|u_{\varepsilon}\right\|_{L^{2}\left(I ; L^{2}(\omega)\right)}^{2}+\left\langle\mathbf{A} \mathbf{y}_{\varepsilon}+\mathbf{B} u_{\varepsilon}, \mathbf{p}_{\varepsilon}\right\rangle_{L^{2}\left(I ; \mathbf{Y}^{0}\right), L^{2}\left(I ; \mathbf{P}^{1}\right)}=0 .
\end{array}\right.
$$

The optimal control $u_{\varepsilon}$ has the additional regularity

$$
u_{\varepsilon} \in C\left(\bar{I} ; L^{2}(\omega)\right) \text { and } \partial_{t} u_{\varepsilon} \in L^{\infty}\left(I ; L^{2}(\omega)\right) .
$$

If, moreover, $\mathbf{y}_{0} \in \mathbf{Y}^{2}$, then

$$
\mathbf{y}_{\varepsilon} \in C\left(\bar{I} ; \mathbf{Y}^{2}\right) \cap C^{1}\left(\bar{I} ; \mathbf{Y}^{1}\right) .
$$

An interesting and not yet completely answered question is under which conditions one can pass to the limit $\varepsilon \searrow 0$ in the above optimality condition. Here, we have the following result.

TheOREM 2.5. Let $\left\{\left(\tau_{\varepsilon}, \mathbf{y}_{\varepsilon}, u_{\varepsilon}\right)\right\}_{\varepsilon>0}$ be solutions of $\left(P_{\varepsilon}\right)$ and $\left\{\mathbf{p}_{\varepsilon}\right\}_{\varepsilon>0}$ such that (2.5) is satisfied. Let (H1) be satisfied, such that the wave equation (2.2) is controllable for $\tau_{0}<\tau^{*}$. Let $(\tilde{\mathbf{y}}, \tilde{u})$ be a weak-star accumulation point of $\left\{\left(\mathbf{y}_{\varepsilon}, u_{\varepsilon}\right)\right\}$ as in Theorem 2.3 such that $\tilde{u}$ is bang-bang, i.e., $\|u(t)\|_{L^{2}(\omega)}=\gamma$ a.e. on $I$. 
Then there is a nontrivial $\tilde{\mathbf{p}} \in C\left(\bar{I} ; \mathbf{P}^{1}\right)$ such that

$$
\left\{\begin{array}{l}
\tilde{\mathbf{y}}_{t}=\tau^{*} \mathbf{A} \tilde{\mathbf{y}}+\tau^{*} \mathbf{B} \tilde{u}, \tilde{\mathbf{y}}(0)=\mathbf{y}_{0}, \tilde{\mathbf{y}}(1)=\mathbf{z}, \\
-\tilde{\mathbf{p}}_{t}=\tau^{*} \mathbf{A}^{*} \tilde{\mathbf{p}}, \\
\left(\mathbf{B}^{*} \tilde{\mathbf{p}}, u-\tilde{u}\right)_{L^{2}\left(I ; L^{2}(\omega)\right)} \geq 0 \text { for all } u \in U_{a d} .
\end{array}\right.
$$

If the sequence $\left\{\mathbf{p}_{\varepsilon}(1)\right\}$ is bounded in $\mathbf{P}^{1}$, then

$$
1+\langle\mathbf{A z}+\mathbf{B} \tilde{u}(1), \tilde{\mathbf{p}}\rangle_{\mathbf{Y}^{1}, \mathbf{P}^{0}}=0 .
$$

If the sequence $\left\{\mathbf{p}_{\varepsilon}(1)\right\}$ is unbounded in $\mathbf{P}^{0}$ and $\mathbf{B}^{*} \tilde{\mathbf{p}}(1) \neq 0$, then $\mathbf{z} \neq 0$ and it holds

$$
\langle\mathbf{A z}+\mathbf{B} \tilde{u}(1), \tilde{\mathbf{p}}\rangle_{\mathbf{Y}^{1}, \mathbf{P}^{0}}=0 .
$$

Proof. For the proof we refer to [19, Thm. 4.4 and Cor. 4.6].

The necessity of condition (2.6) was proved by Fattorini [5]. If $\omega=\Omega$, this implies the bang-bang property of $\tilde{u}$, i.e., $\|u(t)\|_{L^{2}(\omega)}=\gamma$ for all but finitely many $t \in[0,1]$. It is still open whether this bang-bang property holds for the general case $\omega \neq \Omega$. Additionally, it is open if it is necessary for optimality that one of the equations (2.7) or (2.8) holds. Hence, we will report on the boundedness of $\left\{\mathbf{p}_{\varepsilon}(1)\right\}$ or lack thereof in the evaluation of the numerical results in section 5.2 in order to argue with Theorem 2.5 that (2.7) or (2.8) holds.

3. Sensitivity analysis for a parametric optimization problem. Throughout this section we fix $\varepsilon>0$ and consider for any $\tau>0$ the minimization problem with respect to the variable $u$ :

$\left(P_{\varepsilon}^{\tau}\right)$

$$
\left\{\begin{array}{l}
\min _{u \in U_{a d}} \tau\left(1+\frac{\varepsilon}{2}\|u\|_{L^{2}\left(I ; L^{2}(\omega)\right)}^{2}\right)+\frac{1}{2 \varepsilon}\|\mathbf{y}(1)-\mathbf{z}\|_{\mathbf{Y}^{0}}^{2} \\
\text { subject to } \\
\mathbf{y}_{t}=\tau \mathbf{A y}+\tau \mathbf{B} u \text { on }(0,1], \\
\mathbf{y}(0)=\mathbf{y}_{0} .
\end{array}\right.
$$

Clearly this problem admits a unique solution $\left(\mathbf{y}_{\tau}, u_{\tau}\right) \in\left(C\left(\bar{I} ; \mathbf{Y}^{1}\right) \cap C^{1}\left(\bar{I} ; \mathbf{Y}^{0}\right)\right) \times$ $L^{2}\left(I ; L^{2}(\omega)\right)$, and the necessary and sufficient optimality condition is given by

$$
\left\{\begin{array}{l}
\partial_{t} \mathbf{y}_{\tau}=\tau \mathbf{A y}_{\tau}+\tau \mathbf{B} u_{\tau}, \quad \mathbf{y}_{\tau}(0)=\mathbf{y}_{0}, \\
-\partial_{t} \mathbf{p}_{\tau}=\tau \mathbf{A}^{*} \mathbf{p}_{\tau}, \mathbf{p}_{\tau}(1)=\frac{1}{\varepsilon}\left(\begin{array}{c}
\mathbf{y}_{\tau, 1}(1)-z_{1} \\
(-\Delta)^{-1}\left(\mathbf{y}_{\tau, 2}(1)-z_{2}\right)
\end{array}\right), \\
\left(\varepsilon u_{\tau}+\mathbf{B}^{*} \mathbf{p}_{\tau}, u-u_{\tau}\right)_{L^{2}\left(I ; L^{2}(w)\right)} \geq 0 \text { for all } u \in U_{a d} .
\end{array}\right.
$$

Here we use the index $\tau$ for the solution to $\left(P_{\varepsilon}^{\tau}\right)$, since our attention focuses on the dependence of the solution on this parameter. We will investigate differentiability of $\tau \rightarrow\left(\mathbf{y}_{\tau}, u_{\tau}, \mathbf{p}_{\tau}\right)$ as well as first- and second-order differentiability of the value functional in $\left(P_{\varepsilon}^{\tau}\right)$ with respect to $\tau$. Further we shall show that the derivative of this value function is given by the left-hand side of the transversality condition, which is the last equation in (2.5). Some preliminaries will be required. Let us note that these results apparently cannot be directly derived from well-known abstract sensitivity results. This is due to the fact that these results require us to express the constraint in an abstract setting, in which the partial differential equation in $\left(P_{\varepsilon}^{\tau}\right)$ is transformed to 
an equality constraint of the form $e(y, u)=0$. The natural domain for $e$ is $\left(L^{2}\left(I ; \mathbf{Y}^{1}\right) \cap\right.$ $\left.H^{1}\left(I ; \mathbf{Y}^{0}\right) \cap\left\{\mathbf{y}:\left(y_{2}\right)_{t}-\tau \Delta y_{1} \in L^{2}(\Omega)\right\}\right) \times L^{2}\left(I ; L^{2}(\omega)\right)$, which is a Banach space when endowed with the natural norm. However, this space depends on the the parameter $\tau$. For this reason and due to the fact that the image space of the inequality constraints is infinite dimensional, general results appear not to be readily applicable. In this section we do not need to require (H1) to hold, but throughout we assume

$$
\mathbf{y}_{0} \in \mathbf{Y}^{2} .
$$

With (H2) holding the solution to $\left(P_{\varepsilon}^{\tau}\right)$ satisfies the regularity

$$
\mathbf{y}_{\tau} \in C\left(\bar{I} ; \mathbf{Y}^{2}\right) \cap C^{1}\left(\bar{I} ; \mathbf{Y}^{1}\right) .
$$

This can be verified with the same techniques that provide the regularity of $\mathbf{y}_{\varepsilon}$ in Theorem 2.4.

Proposition 3.1. Let (H2) hold. Then for every compact subset $J \subset(0, \infty)$ the set $\left\{\left(\mathbf{y}_{\tau}, u_{\tau}, \mathbf{p}_{\tau}\right): \tau \in J\right\}$ is bounded in $\left(C\left(\bar{I} ; \mathbf{Y}^{2}\right) \cap C^{1}\left(\bar{I} ; \mathbf{Y}^{1}\right)\right) \times W^{1, \infty}\left(I ; L^{2}(\omega)\right) \times$ $\left(C\left(\bar{I} ; \mathbf{P}^{2}\right) \cap C^{1}\left(\bar{I} ; \mathbf{P}^{1}\right)\right)$ and the mapping $\tau \rightarrow\left(\mathbf{y}_{\tau}, u_{\tau}, \mathbf{p}_{\tau}\right)$ is globally Lipschitz continuous from $J$ to $\left(C\left(\bar{I} ; \mathbf{Y}^{1}\right) \cap W^{1,2}\left(I ; \mathbf{Y}^{0}\right)\right) \times L^{2}\left(I ; L^{2}(\omega)\right) \times\left(C\left(\bar{I} ; \mathbf{P}^{1}\right) \cap W^{1,2}\left(I ; \mathbf{P}^{0}\right)\right)$.

Proof. We first show the asserted uniform bound. Since the set $\left\{u_{\tau}: \tau \in J\right\}$ is bounded in $L^{2}\left(I ; L^{2}(\omega)\right)$ it follows that $\left\{\mathbf{y}_{\tau}: \tau \in J\right\}$ is bounded in $C\left(\bar{I} ; \mathbf{Y}^{1}\right) \cap$ $W^{1,2}\left(I ; \mathbf{Y}^{0}\right)$ and $\left\{\mathbf{p}_{\tau}: \tau \in J\right\}$ is bounded in $C\left(\bar{I} ; \mathbf{P}^{2}\right) \cap C^{1}\left(\bar{I} ; \mathbf{P}^{1}\right)$. Using that $u_{\tau}=$ $-P_{U_{a d}}\left(\frac{1}{\varepsilon} \mathbf{p}_{\tau, 2}\right)$ it follows from Lemma 3.2 below that $\left\{u_{\tau}: \tau \in J\right\}$ is bounded in $W^{1, \infty}\left(I ; L^{2}(\omega)\right)$. This, together with $\mathbf{y}_{0} \in \mathbf{Y}^{2}$, implies that $\left\{\mathbf{y}_{\tau}: \tau \in J\right\}$ is bounded in $C\left(\bar{I} ; \mathbf{Y}^{2}\right) \cap C^{1}\left(\bar{I} ; \mathbf{Y}^{1}\right)$.

Let $\bar{\tau} \in J$ and $\tau \in J$ and set

$$
(\delta \tau, \boldsymbol{\delta} \mathbf{y}, \delta u, \delta \mathbf{p})=\left(\bar{\tau}-\tau, \mathbf{y}_{\bar{\tau}}-\mathbf{y}_{\tau}, u_{\bar{\tau}}-u_{\tau}, \mathbf{p}_{\bar{\tau}}-\mathbf{p}_{\tau}\right),
$$

where $\left(\mathbf{y}_{\tau}, u_{\tau}, \mathbf{p}_{\tau}\right)$ is the solution to $\left(P_{\varepsilon}^{\tau}\right)$ and analogously for $\left(\mathbf{y}_{\bar{\tau}}, u_{\bar{\tau}}, \mathbf{p}_{\bar{\tau}}\right)$. Then we have

$$
\left\{\begin{array}{l}
\partial_{t} \boldsymbol{\delta} \mathbf{y}=\tau(\mathbf{A} \boldsymbol{\delta} \mathbf{y}+\mathbf{B} \delta u)+\delta \tau\left(\mathbf{A y}_{\bar{\tau}}+\mathbf{B} u_{\bar{\tau}}\right), \boldsymbol{\delta} \mathbf{y}(0)=0 \\
-\partial_{t} \boldsymbol{\delta} \mathbf{p}=\tau \mathbf{A}^{*} \boldsymbol{\delta} \mathbf{p}+\delta \tau \mathbf{A}^{*} \mathbf{p}_{\bar{\tau}}, \boldsymbol{\delta} \mathbf{p}(1)=\frac{1}{\varepsilon}\left(\begin{array}{c}
\delta \mathbf{y}_{1}(1) \\
(-\Delta)^{-1} \boldsymbol{\delta} \mathbf{y}_{2}(1)
\end{array}\right) \\
\left(\varepsilon u_{\tau}+\mathbf{B}^{*} \mathbf{p}_{\tau}, u-u_{\tau}\right)_{L^{2}\left(I ; L^{2}(w)\right) \geq 0}, \\
\left(\varepsilon u_{\bar{\tau}}+\mathbf{B}^{*} \mathbf{p}_{\bar{\tau}}, u-u_{\bar{\tau}}\right)_{L^{2}\left(I ; L^{2}(w)\right)} \geq 0 \text { for all } u \in U_{a d} .
\end{array}\right.
$$

We note that the scalar form of the first equation in (3.3) is given by

$$
\left\{\begin{array}{l}
\partial_{t t} \boldsymbol{\delta} \mathbf{y}_{1}=\tau^{2}\left(\Delta \delta \mathbf{y}_{1}+\chi_{\omega} \delta u\right)+\tau \delta \tau\left(\Delta \mathbf{y}_{\bar{\tau}, 1}+\chi_{\omega} u_{\bar{\tau}}\right)+\delta \tau \partial_{t} \mathbf{y}_{\bar{\tau}, 2} \\
\delta \mathbf{y}_{1}(0)=0, \partial_{t} \delta \mathbf{y}_{1}(0)=\delta \tau y_{2}
\end{array}\right.
$$

and for the second component we have $\tau \boldsymbol{\delta} \mathbf{y}_{2}=\partial_{t} \boldsymbol{\delta} \mathbf{y}_{1}-\delta \tau \mathbf{y}_{\bar{\tau}, 2}$. Similarly for the second equation in (3.3) we find

$$
\left\{\begin{array}{l}
\partial_{t t} \boldsymbol{\delta} \mathbf{p}_{2}=\tau^{2} \Delta \delta \mathbf{p}_{2}+\tau \delta \tau \Delta \mathbf{p}_{\bar{\tau}, 2}-\delta \tau \partial_{t} \mathbf{p}_{\bar{\tau}, 1} \\
\delta \mathbf{p}_{2}(1)=\frac{1}{\varepsilon}(-\Delta)^{-1} \delta \mathbf{y}_{2}(1), \partial_{t} \boldsymbol{\delta} \mathbf{p}_{2}(1)=-\frac{\tau}{\varepsilon} \boldsymbol{\delta} \mathbf{y}_{1}(1)-\frac{\delta \tau}{\varepsilon}\left(\mathbf{y}_{\varepsilon, 1}(1)-z_{1}\right)
\end{array}\right.
$$

and for the first component we have $\tau \boldsymbol{\delta} \mathbf{p}_{1}=-\partial_{t} \boldsymbol{\delta} \mathbf{p}_{2}-\delta \tau \mathbf{p}_{\bar{\tau}, 1}$. 
From Theorem 2.1, the first two equations in (3.3), and (3.4), (3.5) there exists a constant $K_{1}$ independent of $\tau \in J$ such that

$$
\left\{\begin{array}{l}
\|\boldsymbol{\delta} \mathbf{y}\|_{C\left(\bar{I} ; \mathbf{Y}^{1}\right) \cap W^{1,2}\left(I ; \mathbf{Y}^{0}\right)} \leq K_{1}\left(|\delta \tau|+\|\delta u\|_{L^{2}\left(I ; L^{2}(\omega)\right)}\right), \\
\|\boldsymbol{\delta} \mathbf{p}\|_{C\left(\bar{I} ; \mathbf{P}^{1}\right) \cap W^{1,2}\left(I ; \mathbf{P}^{0}\right)} \leq K_{1}\left(|\delta \tau|+\|\boldsymbol{\delta} \mathbf{y}(1)\|_{\mathbf{Y}^{0}}\right) .
\end{array}\right.
$$

Setting $u=u_{\bar{\tau}}$ and $u=u_{\tau}$ in the two inequalities of (3.3) we obtain, after rearranging terms,

$$
\varepsilon\|\delta u\|_{L^{2}\left(I ; L^{2}(\omega)\right.}^{2} \leq-\left(\mathbf{B}^{*} \delta \mathbf{p}, \delta u\right)_{L^{2}\left(I ; L^{2}(\omega)\right)} .
$$

Next we take the inner product in $L^{2}\left(I ; L^{2}(\Omega)\right)$ of the first equation in (3.1) with $\delta \mathbf{p}$ and of the second equation with $\delta \mathbf{y}$. After integration by parts and subtraction of the two resulting equations we obtain, using that $\boldsymbol{\delta} \mathbf{y}(0)=0$,

$$
(\boldsymbol{\delta} \mathbf{y}(1), \delta \mathbf{p}(1))_{L^{2}(\Omega)}=\tau(\mathbf{B} \delta u, \boldsymbol{\delta} \mathbf{p})+\delta \tau\left(\left(\mathbf{A y}_{\bar{\tau}}+\mathbf{B} u_{\bar{\tau}}, \boldsymbol{\delta} \mathbf{p}\right)-\left(\mathbf{A}^{*} \mathbf{p}_{\bar{\tau}}, \boldsymbol{\delta} \mathbf{y}\right)\right),
$$

where the inner products on the right-hand side are taken in $L^{2}\left(I ; L^{2}(\Omega)\right)$. The initial condition for $\boldsymbol{\delta} \mathbf{p}(1)$ and (3.7) imply that

$$
\frac{1}{\varepsilon}\|\boldsymbol{\delta} \mathbf{y}(1)\|_{\mathbf{Y}^{0}}^{2}+\tau \varepsilon\|\delta u\|_{L^{2}\left(I ; L^{2}(\omega)\right)}^{2} \leq \delta \tau\left(\left|\left(\mathbf{A y}_{\bar{\tau}}+\mathbf{B} u_{\bar{\tau}}, \boldsymbol{\delta} \mathbf{p}\right)\right|+\left|\left(\mathbf{A}^{*} \mathbf{p}_{\bar{\tau}}, \boldsymbol{\delta} \mathbf{y}\right)\right|\right) .
$$

Using (3.6) and the estimates established at the beginning of the proof, we obtain the existence of a constant $K_{2}$ independent of $\tau \in J, \bar{\tau} \in J$ such that

$$
\frac{1}{\varepsilon}\|\boldsymbol{\delta} \mathbf{y}(1)\|_{\mathbf{Y}^{0}}^{2}+\tau \varepsilon\|\delta u\|_{L^{2}\left(I ; L^{2}(\omega)\right)}^{2} \leq K_{2}|\delta \tau|\left(\|\delta u\|_{L^{2}\left(I ; L^{2}(\omega)\right)}+|\delta \tau|\right),
$$

which, in turn, implies the existence of a constant $K_{3}$ independent of $\tau \in J, \bar{\tau} \in J$, such that

$$
\frac{1}{\varepsilon}\|\boldsymbol{\delta} \mathbf{y}(1)\|_{\mathbf{Y}^{0}}^{2}+\varepsilon\|\delta u\|_{L^{2}\left(I ; L^{2}(\omega)\right)}^{2} \leq K_{3}|\delta \tau|^{2} .
$$

The conclusion now follows from (3.6).

Let us now turn to a continuity result for the projection onto the set of admissible controls. By construction of $U_{a d}$, we have

$$
\left(P_{U_{a d}}(u)\right)(t):=P_{U}(u(t)) \text { for almost all (f.a.a.) } t \in(0,1),
$$

where the projection onto $U$ is defined as

$$
P_{U}(u)=\min \left(1, \frac{\gamma}{\|u\|_{L^{2}(\omega)}}\right) .
$$

LEMma 3.2. The projection $P_{U_{a d}}$ is continuous mapping from $C^{1}\left(\bar{I} ; L^{2}(\omega)\right)$ to $H^{1}\left(I ; L^{2}(\omega)\right)$.

Proof. The proof can be found in the appendix; see section 7 .

This continuity result for the projection onto the set of admissible controls allows one to conclude continuity of the mapping $\tau \mapsto\left(\mathbf{y}_{\tau}, u_{\tau}, \mathbf{p}_{\tau}\right)$ with respect to stronger norms.

TheOrem 3.3. Let (H2) hold. Then the mapping $\tau \rightarrow\left(\mathbf{y}_{\tau}, u_{\tau}, \mathbf{p}_{\tau}\right)$ is continuous at $\tau>0$ with values in $\left(C\left(\bar{I} ; \mathbf{Y}^{2}\right) \cap H^{1}\left(I ; \mathbf{Y}^{1}\right)\right) \times H^{1}\left(I ; L^{2}(\omega)\right) \times\left(C\left(\bar{I} ; \mathbf{P}^{2}\right) \cap H^{1}\left(I ; \mathbf{P}^{2}\right)\right)$.

Copyright $@$ by SIAM. Unauthorized reproduction of this article is prohibited. 
Proof. Due to the result of Proposition 3.1, we have the Lipschitz continuity of $\tau \mapsto \mathbf{p}_{\tau}$ with values in $C\left(\bar{I} ; \mathbf{P}^{1}\right)$. This implies the Lipschitz continuity of $\tau \mapsto$ $\mathbf{B}^{*} \mathbf{p}_{\tau}$ with values in $C^{1}\left(\bar{I} ; L^{2}(\omega)\right)$. Hence, by Lemma 3.2 the mapping $\tau \mapsto u_{\tau}=$ $P_{U_{a d}}\left(-\frac{1}{\varepsilon} \mathbf{B}^{*} \mathbf{p}_{\tau}\right)$ is continuous with values in $H^{1}\left(\bar{I} ; L^{2}(\omega)\right)$. The result now follows with the regularity result of Theorem 2.1.

We will now prove directional differentiability of the mapping $\tau \mapsto\left(\mathbf{y}_{\tau}, u_{\tau}, \mathbf{p}_{\tau}\right)$ directly, using the projection representation $u_{\tau}=P_{U_{a d}}\left(-\frac{1}{\varepsilon} \mathbf{B}^{*} \mathbf{p}_{\tau}\right)$, following the lines of a similar result in [7]. Since $\left(P_{\varepsilon}^{\tau}\right)$ is an inequality-constrained optimal control problem, we can expect directional differentiability of the mapping $\tau \mapsto\left(\mathbf{y}_{\tau}, u_{\tau}, \mathbf{p}_{\tau}\right)$. It turns out that the concept of Bouligand differentiability is suitable for the subsequent analysis.

First, we will show Bouligand differentiability of the projection onto $U_{a d}$. Recall that a function $f: X \rightarrow Y$ between normed linear spaces $X$ and $Y$ is called Bouligand differentiable if for every $x_{0} \in X$ there exists $\varepsilon>0$ and a positively homogeneous function $f^{\prime}\left(x_{0} ; \cdot\right): X \rightarrow Y$ such that

$$
f(x)=f\left(x_{0}\right)+f^{\prime}\left(x_{0} ; x-x_{0}\right)+r\left(x_{0} ; x-x_{0}\right)
$$

for all $x \in X$, where $\left\|r\left(x_{0} ; x-x_{0}\right)\right\|_{Y} /\left\|x-x_{0}\right\|_{X} \rightarrow 0$ for $\left\|x-x_{0}\right\|_{X} \rightarrow 0$. In particular this implies that $f$ is directionally differentiable.

Proposition 3.4. The projection $P_{U}$ onto $U$, where $U$ is given by $U=\{u \in$ $\left.L^{2}(\omega):\|u\|_{L^{2}(\omega)} \leq \gamma\right\}$, is Bouligand differentiable from $L^{2}(\omega)$ to $L^{2}(\omega)$. That is, for all $q, h \in L^{2}(\omega)$ we have

$$
\left\|P_{U}(q+h)-P_{U}(q)-P_{U}^{\prime}(q ; h)\right\|_{L^{2}(\omega)}=o\left(\|h\|_{L^{2}(\omega)}\right)
$$

uniformly on bounded subsets of $L^{2}(\omega)$, where the Bouligand derivative is given by

$$
P_{U}^{\prime}(q ; h)= \begin{cases}h & \text { if }\|q\|_{L^{2}(\omega)}<\gamma, \\ \gamma\left(\frac{h}{\|q\|_{L^{2}(\omega)}}-\frac{(q, h)_{L^{2}(\omega)}}{\|q\|_{L^{2}(\omega)}^{3}} q\right) & \text { or }\|q\|_{L^{2}(\omega)}=\gamma,(q, h)<0, \\ \text { if }\|q\|_{L^{2}(\omega)}>\gamma, & \text { or }\|q\|_{L^{2}(\omega)}=\gamma,(q, h) \geq 0 .\end{cases}
$$

Proof. The proof can be found in the appendix, section 7 .

We note that $P_{U}^{\prime}(q ; h)$ is not linear in $h$. Hence $P_{U}$ is not Gateaux differentiable. Due to the dependence on the direction the Bouligand derivative is not well suited for numerical realizations. For this purpose we shall use the Newton derivative in section 4 .

It can also be noted that the directional derivative $P_{U}^{\prime}$ is itself a projection onto a convex set:

$$
P_{U}^{\prime}(q ; h)=P_{T_{U}(q)}(h)
$$

with

$$
T_{U}(q):= \begin{cases}L^{2}(\omega) & \text { if }\|q\|_{L^{2}(\omega)}<\gamma, \\ \left\{h \in L^{2}(\Omega):(h, q) \leq 0\right\} & \text { if }\|q\|_{L^{2}(\omega)}=\gamma, \\ \left\{h \in L^{2}(\Omega):(h, q)=0\right\} & \text { if }\|q\|_{L^{2}(\omega)}>\gamma .\end{cases}
$$

Proposition 3.5. The projection $P_{U_{a d}}$ is Bouligand differentiable from $C\left(\bar{I} ; L^{2}(\omega)\right)$ to $L^{2}\left(I ; L^{2}(\omega)\right)$ with directional derivative

$$
\left(P_{U_{a d}}^{\prime}(q ; h)\right)(t)=P_{U}^{\prime}(q(t) ; h(t)) \quad \text { for all } t \in \bar{I} .
$$

Copyright (c) by SIAM. Unauthorized reproduction of this article is prohibited. 
Proof. The proof can be found in the appendix, section 7 .

TheOREM 3.6. Let (H2) hold. Then the mapping $\tau \mapsto\left(\mathbf{y}_{\tau}, u_{\tau}, \mathbf{p}_{\tau}\right)$ is Bouliganddifferentiable from $\mathbb{R}^{+}$to $C\left(\bar{I} ; \mathbf{Y}^{1}\right) \times L^{2}\left(I ; L^{2}(\omega)\right) \times C\left(\bar{I} ; \mathbf{P}^{1}\right)$. The directional derivative in direction $\delta \tau$ is given as the unique solution $(\dot{\mathbf{y}}, \dot{u}, \dot{\mathbf{p}}) \in C\left(\bar{I} ; \mathbf{Y}^{1}\right) \times L^{2}\left(I ; L^{2}(\omega)\right) \times$ $C\left(\bar{I} ; \mathbf{P}^{1}\right)$ of the system

$$
\left\{\begin{array}{l}
\partial_{t} \dot{\mathbf{y}}=\tau(\mathbf{A} \dot{\mathbf{y}}+\mathbf{B} \dot{u})+\delta \tau\left(\mathbf{A y}_{\tau}+\mathbf{B} u_{\tau}\right), \dot{\mathbf{y}}(0)=0 \\
-\partial_{t} \dot{\mathbf{p}}=\tau \mathbf{A}^{*} \dot{\mathbf{p}}+\delta \tau \mathbf{A p}_{\tau}, \dot{\mathbf{p}}(1)=\frac{1}{\varepsilon}\left(\begin{array}{c}
\dot{\mathbf{y}}_{1}(1) \\
(-\Delta)^{-1} \dot{\mathbf{y}}_{2}(1)
\end{array}\right), \\
\dot{u} \in T_{U_{a d}\left(-\frac{1}{\epsilon} \mathbf{B}^{*} \mathbf{p}_{\tau}\right)}, \\
\left(\varepsilon \dot{u}+\mathbf{B}^{*} \dot{\mathbf{p}}, v-\dot{u}\right)_{L^{2}\left(I ; L^{2}(\omega)\right)} \geq 0 \quad \text { for all } v \in T_{U_{a d}\left(-\frac{1}{\epsilon} \mathbf{B}^{*} \mathbf{p}_{\tau}\right)},
\end{array}\right.
$$

where $T_{U_{a d}\left(-\frac{1}{\epsilon} \mathbf{B}^{*} \mathbf{p}_{\tau}\right)}$ is given by

$$
T_{U_{a d}\left(-\frac{1}{\epsilon} \mathbf{B}^{*} \mathbf{p}_{\tau}\right)}=\left\{u \in L^{2}\left(I ; L^{2}(\omega)\right): u(t) \in T_{U}\left(-\frac{1}{\epsilon} \mathbf{B}^{*} \mathbf{p}_{\tau}(t)\right) \text { a.e. on } I\right\}
$$

with $T_{U}$ defined in (3.9).

Proof. Let us define for abbreviation $T:=T_{U_{a d}\left(-\frac{1}{\epsilon} \mathbf{B}^{*} \mathbf{p}_{\tau}\right)}$.

Let $\tau>0, \delta \tau \in(-1,1)$ with $\tau+\delta \tau>0$ be given. At first, let us prove the existence and uniqueness of solutions of the sensitivity system (3.10). This system is the first-order necessary optimality condition of the optimization problem

$$
\min \tau \frac{\varepsilon}{2}\|u\|_{L^{2}\left(I ; L^{2}(\omega)\right)}^{2}+\frac{1}{2 \varepsilon}\|\mathbf{y}(1)\|_{\mathbf{Y}^{1}}^{2}+\delta \tau\left\langle\mathbf{A}^{*} \mathbf{p}_{\tau}, \mathbf{y}\right\rangle_{L^{2}\left(I ; \mathbf{P}^{1}\right), L^{2}\left(I ; \mathbf{Y}^{0}\right)}
$$

subject to $u \in T$ and

$$
\partial_{t} \mathbf{y}=\tau(\mathbf{A y}+\mathbf{B} u)+\delta \tau\left(\mathbf{A} \mathbf{y}_{\tau}+\mathbf{B} u_{\tau}\right), \mathbf{y}(0)=0 .
$$

Since this problem is strictly convex with respect to $u$, it admits a unique solution. This proves that there exists a unique solution $(\dot{\mathbf{y}}, \dot{u}, \dot{\mathbf{p}})$ of the system (3.10).

Let us define

$$
(\boldsymbol{\delta} \mathbf{y}, \delta u, \delta \mathbf{p})=\left(\mathbf{y}_{\tau+\delta \tau}-\mathbf{y}_{\tau}, u_{\tau+\delta \tau}-u_{\tau}, \mathbf{p}_{\tau+\delta \tau}-\mathbf{p}_{\tau}\right) .
$$

Then $(\boldsymbol{\delta} \mathbf{y}, \delta u, \delta \mathbf{p})$ solves the system

$$
\left\{\begin{array}{l}
\partial_{t} \boldsymbol{\delta} \mathbf{y}=\tau(\mathbf{A} \boldsymbol{\delta} \mathbf{y}+\mathbf{B} \delta u)+\delta \tau\left(\mathbf{A} \mathbf{y}_{\tau+\delta \tau}+\mathbf{B} u_{\tau+\delta \tau}\right), \boldsymbol{\delta} \mathbf{y}(0)=0 \\
-\partial_{t} \boldsymbol{\delta} \mathbf{p}=\tau \mathbf{A}^{*} \boldsymbol{\delta} \mathbf{p}+\delta \tau \mathbf{A}^{*} \mathbf{p}_{\tau+\delta \tau}, \boldsymbol{\delta} \mathbf{p}(1)=\frac{1}{\varepsilon}\left(\begin{array}{c}
\boldsymbol{\delta} \mathbf{y}_{1}(1) \\
(-\Delta)^{-1} \delta \mathbf{y}_{2}(1)
\end{array}\right) \\
\delta u=P_{U_{a d}}\left(-\frac{1}{\varepsilon} \mathbf{B}^{*} \mathbf{p}_{\tau+\delta \tau}\right)-P_{U_{a d}}\left(-\frac{1}{\varepsilon} \mathbf{B}^{*} \mathbf{p}_{\tau}\right)
\end{array}\right.
$$

By the result of Proposition 3.5, we have

$$
\delta u=P_{U_{a d}}\left(-\frac{1}{\varepsilon} \mathbf{B}^{*} \mathbf{p}_{\tau+\delta \tau}\right)-P_{U_{a d}}\left(-\frac{1}{\varepsilon} \mathbf{B}^{*} \mathbf{p}_{\tau}\right)=P_{T}\left(-\frac{1}{\varepsilon} \mathbf{B}^{*} \delta \mathbf{p}\right)+r
$$

with $\|r\|_{L^{2}\left(I ; L^{2}(\omega)\right)}=o\left(\|\boldsymbol{\delta} \mathbf{p}\|_{C\left(\bar{I} ; \mathbf{P}^{0}\right)}\right)$ as $\|\boldsymbol{\delta} \mathbf{p}\|_{C\left(\bar{I} ; \mathbf{P}^{0}\right)} \rightarrow 0$. With Proposition 3.1 we have $\|r\|_{L^{2}\left(I ; L^{2}(\omega)\right)}=o(|\delta \tau|)$. Let us write this representation of $\delta u$ as the variational inequality

$$
\left(\varepsilon(\delta u-r)+\mathbf{B}^{*} \delta \mathbf{p}, v-(\delta u-r)\right)_{L^{2}\left(I ; L^{2}(\omega)\right)} \geq 0 \quad \text { for all } v \in T .
$$

Copyright $@$ ㅇ by SIAM. Unauthorized reproduction of this article is prohibited. 
Setting $v:=\dot{u}$ in this inequality and $v:=\delta u-r$ in the fourth relation of (3.10) and adding the resulting inequalities gives

$\varepsilon\|\dot{u}-\delta u+r\|_{L^{2}\left(I ; L^{2}(\omega)\right)}^{2} \leq\left(\mathbf{B}^{*}(\dot{\mathbf{p}}-\boldsymbol{\delta} \mathbf{p}), \delta u-\dot{u}\right)_{L^{2}\left(I ; L^{2}(\omega)\right)}-\left(\mathbf{B}^{*}(\dot{\mathbf{p}}-\boldsymbol{\delta} \mathbf{p}), r\right)_{L^{2}\left(I ; L^{2}(\omega)\right)}$,

which implies

$$
\begin{aligned}
\frac{\varepsilon}{2}\|\dot{u}-\delta u\|_{L^{2}\left(I ; L^{2}(\omega)\right)}^{2} \leq & \varepsilon\|r\|_{L^{2}\left(I ; L^{2}(\omega)\right)}^{2} \\
& -\left(\mathbf{B}^{*}(\dot{\mathbf{p}}-\boldsymbol{\delta} \mathbf{p}), r\right)_{L^{2}\left(I ; L^{2}(\omega)\right)} \\
& +\left(\mathbf{B}^{*}(\dot{\mathbf{p}}-\boldsymbol{\delta} \mathbf{p}), \delta u-\dot{u}\right)_{L^{2}\left(I ; L^{2}(\omega)\right)}
\end{aligned}
$$

Proceeding as in the proof of Proposition 3.1 to estimate the last term we find

$$
\begin{aligned}
\tau \frac{\varepsilon}{2} \| & \dot{u}-\delta u\left\|_{L^{2}\left(I ; L^{2}(\omega)\right)}^{2}+\frac{1}{\varepsilon}\right\| \dot{\mathbf{y}}(1)-\delta \mathbf{y}(1) \|_{\mathbf{Y}^{0}}^{2} \\
\leq & \tau\|r\|_{L^{2}\left(I ; L^{2}(\omega)\right)}^{2}-\tau\left(\mathbf{B}^{*}(\dot{\mathbf{p}}-\delta \mathbf{p}), r\right)_{L^{2}\left(I ; L^{2}(\omega)\right)} \\
& +\delta \tau\langle\mathbf{A} \delta \mathbf{y}+\mathbf{B} \delta u, \dot{\mathbf{p}}-\boldsymbol{\delta} \mathbf{p}\rangle_{L^{2}\left(I ; \mathbf{Y}^{0}\right), L^{2}\left(I ; \mathbf{P}^{1}\right)} \\
& +\delta \tau\left\langle\mathbf{A}^{*} \boldsymbol{\delta} \mathbf{p}, \dot{\mathbf{y}}-\boldsymbol{\delta} \mathbf{y}\right\rangle_{L^{2}\left(I ; \mathbf{P}^{0}\right), L^{2}\left(I ; \mathbf{Y}^{1}\right)}
\end{aligned}
$$

We shall return to this estimate below. Now we introduce $\mathbf{w}=\boldsymbol{\delta} \mathbf{y}-\dot{\mathbf{y}}$ and note that

$$
\partial_{t} \mathbf{w}=\tau(\mathbf{A w}+\mathbf{B}(\delta u-\dot{u}))+\delta \tau(\mathbf{A} \delta \mathbf{y}+\mathbf{B} \delta u), \delta \mathbf{w}(0)=0 .
$$

As in the proof of Proposition 3.1, see (3.4), we next write this equation in its coordinates:

$$
\left\{\begin{array}{l}
\partial_{t t} \mathbf{w}_{1}=\tau^{2}\left(\Delta \mathbf{w}_{1}+\chi_{\omega}(\delta u-\dot{u})\right)+\tau \delta \tau\left(\Delta\left(\delta \mathbf{y}_{1}\right)+\chi_{\omega} \delta u\right)+\delta \tau \partial_{t}(\boldsymbol{\delta} \mathbf{y})_{2} \\
\mathbf{w}_{1}(0)=0, \partial_{t} \mathbf{w}_{1}(0)=0
\end{array}\right.
$$

and

$$
\tau \mathbf{w}_{2}=\partial_{t} \mathbf{w}_{1}-\delta \tau(\boldsymbol{\delta} \mathbf{y})_{2} .
$$

By Theorem 2.1, there is a constant $K>0$ independent of $\delta \tau$ such that

$$
\begin{aligned}
& \left\|\mathbf{w}_{1}\right\|_{C\left(\bar{I} ; H_{0}^{1}(\Omega)\right)}+\left\|\partial_{t} \mathbf{w}_{1}\right\|_{C\left(\bar{I} ; L^{2}(\Omega)\right)} \\
& \leq K\left(\|\delta u-\dot{u}\|_{L^{2}\left(I ; L^{2}(\omega)\right)}\right. \\
& \left.\quad+\delta \tau\left(\left\|(\boldsymbol{\delta} \mathbf{y})_{1}\right\|_{L^{2}\left(I ; H^{2}(\Omega)\right)}+\left\|(\boldsymbol{\delta} \mathbf{y})_{2}\right\|_{H^{1}\left(I ; L^{2}(\Omega)\right)}+\|\delta u\|_{L^{2}\left(I ; L^{2}(\omega)\right)}\right)\right) .
\end{aligned}
$$

This further implies that

$$
\begin{aligned}
\left\|\mathbf{w}_{2}\right\|_{C\left(\bar{I} ; H_{0}^{1}(\Omega)\right)} \leq K( & \|\delta u-\dot{u}\|_{L^{2}\left(I ; L^{2}(\omega)\right)} \\
& \left.+\delta \tau\left(\|\boldsymbol{\delta} \mathbf{y}\|_{L^{2}\left(I ; \mathbf{Y}^{2}\right)}+\|\boldsymbol{\delta} \mathbf{y}\|_{H^{1}\left(I ; \mathbf{Y}^{1}\right)}+\|\delta u\|_{L^{2}\left(I ; L^{2}(\omega)\right)}\right)\right),
\end{aligned}
$$

and hence

$$
\begin{aligned}
\|\boldsymbol{\delta} \mathbf{y}-\dot{\mathbf{y}}\|_{C\left(\bar{I} ; \mathbf{Y}^{1}\right)} & =\|\mathbf{w}\|_{C\left(\bar{I} ; \mathbf{Y}^{1}\right)} \\
\leq & K\left(\|\delta u-\dot{u}\|_{L^{2}\left(I ; L^{2}(\omega)\right)}\right. \\
& \quad+\delta \tau\left(\|\boldsymbol{\delta} \mathbf{y}\|_{L^{2}\left(I ; \mathbf{Y}^{2}\right)}+\|\boldsymbol{\delta} \mathbf{y}\|_{H^{1}\left(I ; \mathbf{Y}^{1}\right)}+\|\delta u\|_{\left.L^{2}\left(I ; L^{2}(\omega)\right)\right)}\right) .
\end{aligned}
$$

Copyright $@$ by SIAM. Unauthorized reproduction of this article is prohibited. 
Applying the continuity result of Theorem 3.3 to the right-hand side results in

$$
\|\delta \mathbf{y}-\dot{\mathbf{y}}\|_{C\left(\bar{I} ; \mathbf{Y}^{1}\right)} \leq K\|\delta u-\dot{u}\|_{L^{2}\left(I ; L^{2}(\omega)\right)}+o(|\delta \tau|)
$$

for $\delta \tau \rightarrow 0$. In an analogous manner we estimate

$$
\begin{aligned}
\|\boldsymbol{\delta} \mathbf{p}-\dot{\mathbf{p}}\|_{C\left(\bar{I} ; \mathbf{P}^{1}\right)} & \leq K\left(\varepsilon^{-1}\|\boldsymbol{\delta} \mathbf{y}-\dot{\mathbf{y}}\|_{C\left(\bar{I} ; \mathbf{Y}^{0}\right)}+\delta \tau\left(\|\boldsymbol{\delta} \mathbf{p}\|_{L^{2}\left(I ; \mathbf{P}^{2}\right)}+\|\boldsymbol{\delta} \mathbf{p}\|_{H^{1}\left(I ; \mathbf{P}^{1}\right)}\right)\right) \\
& \leq K\|\delta u-\dot{u}\|_{L^{2}\left(I ; L^{2}(\omega)\right)}+o(|\delta \tau|)
\end{aligned}
$$

with $K>0$ independent of $\delta \tau$. We continue to estimate the critical expression on the right-hand side of (3.12) by using (3.13), (3.14), and the Lipschitz continuity result of Proposition 3.1:

$$
\begin{aligned}
& \left|\langle\mathbf{A} \boldsymbol{\delta} \mathbf{y}+\mathbf{B} \delta u, \dot{\mathbf{p}}-\boldsymbol{\delta} \mathbf{p}\rangle_{L^{2}\left(I ; \mathbf{Y}^{0}\right), L^{2}\left(I ; \mathbf{P}^{1}\right)}+\left\langle\mathbf{A}^{*} \delta \mathbf{p}, \dot{\mathbf{y}}-\boldsymbol{\delta} \mathbf{y}\right\rangle_{L^{2}\left(I ; \mathbf{P}^{0}\right), L^{2}\left(I ; \mathbf{Y}^{1}\right)}\right| \\
& \leq\left(\|\boldsymbol{\delta} \mathbf{y}\|_{L^{2}\left(I ; \mathbf{Y}^{1}\right)}+\|\delta u\|_{L^{2}\left(I ; L^{2}(\omega)\right)}\right)\|\boldsymbol{\delta} \mathbf{p}-\dot{\mathbf{p}}\|_{L^{2}\left(\bar{I} ; \mathbf{P}^{1}\right)} \\
& \quad+\|\boldsymbol{\delta} \mathbf{p}\|_{L^{2}\left(\bar{I} ; \mathbf{P}^{1}\right)}\|\boldsymbol{\delta} \mathbf{y}-\dot{\mathbf{y}}\|_{L^{2}\left(\bar{I} ; \mathbf{Y}^{1}\right)} \\
& \leq \\
& \quad \leq \mid \delta \tau \tau\|\delta u-\dot{u}\|_{L^{2}\left(I ; L^{2}(\omega)\right)}+o\left(|\delta \tau|^{2}\right)
\end{aligned}
$$

By (3.12), (3.14), (3.15), we get the estimate

$$
\|\dot{u}-\delta u\|_{L^{2}\left(I ; L^{2}(\omega)\right)}^{2} \leq K|\delta \tau|^{2}\|\delta u-\dot{u}\|_{L^{2}\left(I ; L^{2}(\omega)\right)}+o\left(|\delta \tau|^{2}\right)
$$

with $K$ independent of $\delta \tau$ but dependent on $\varepsilon$. This proves $\|\dot{u}-\delta u\|_{L^{2}\left(I ; L^{2}(\omega)\right)}=$ $o(|\delta \tau|)$ as $\delta \tau \rightarrow 0$. Hence $\left\|u_{\tau+\delta \tau}-u_{\tau}-\dot{u}\right\|_{L^{2}\left(I ; L^{2}(\omega)\right)}=o(|\delta \tau|)$ holds, and by (3.13) and (3.14) we obtain analogous estimates for the state and adjoint variables. This ends the proof.

Corollary 3.7. For any $\tau \in(0, \infty)$ and $\delta \tau$ the Bouligand derivative $\dot{u}$ satisfies

$$
\left(\varepsilon u_{\tau}+\mathbf{B}^{*} \mathbf{p}_{\tau}, \dot{u}\right)_{L^{2}\left(I ; L^{2}(\omega)\right)}=0 .
$$

Proof. Setting $u=u_{\tau+\sigma \delta \tau}$ in the first inequality of (3.3), dividing by $\sigma>0$, and taking $\sigma \rightarrow 0^{+}$, we find $\left(\varepsilon u_{\tau}+\mathbf{B}^{*} \mathbf{p}_{\tau}, \dot{u}\right)_{L^{2}\left(I ; L^{2}(\omega)\right)} \geq 0$. From the second inequality with $u_{\tau}$ replaced by $u_{\bar{\tau}}$ and $u=u_{\tau+\sigma \delta \tau}$ it follows that $\left(\varepsilon u_{\tau}+\mathbf{B}^{*} \mathbf{p}_{\tau}, \dot{u}\right)_{L^{2}\left(I ; L^{2}(\omega)\right)} \leq 0$. Combined this implies (3.16).

Let $\mathcal{V}$ denote the value functional associated to $\left(P_{\varepsilon}^{\tau}\right)$, i.e.,

$$
\mathcal{V}(\tau)=\tau\left(1+\frac{\varepsilon}{2}\left\|u_{\tau}\right\|_{L^{2}\left(I ; L^{2}(\omega)\right)}^{2}\right)+\frac{1}{2 \varepsilon}\left\|\mathbf{y}_{\tau}(1)-\mathbf{z}\right\|_{\mathbf{Y}^{0}}^{2}
$$

and set $\mathbf{L}^{2}(\Omega)=L^{2}(\Omega) \times L^{2}(\Omega)$. The following result establishes smoothness of $\mathcal{V}$ and the relationship of $\frac{d}{d \tau} \mathcal{V}(\tau)$ to the transversality condition.

TheOrem 3.8. Let (H1) hold. Then the mapping $\tau \rightarrow \mathcal{V}(\tau)$ is continuously differentiable on $(0, \infty)$ and

$$
\frac{d}{d \tau} \mathcal{V}(\tau)=1+\frac{\varepsilon}{2}\left\|u_{\tau}\right\|_{L^{2}\left(I ; L^{2}(\omega)\right)}^{2}+\left(\mathbf{A} \mathbf{y}_{\tau}+\mathbf{B} u_{\tau}, \mathbf{p}_{\tau}\right)_{L^{2}\left(I ; \mathbf{L}^{2}(\Omega)\right)}
$$

Moreover, it admits a second-order directional derivative given by

$$
\left(\frac{d}{d \tau}\right)^{2} \mathcal{V}(\tau) \delta \tau=\left\langle\mathbf{A} \mathbf{y}_{\tau}+\mathbf{B} u_{\tau}, \dot{\mathbf{p}}\right\rangle_{L^{2}\left(I ; \mathbf{Y}^{0}\right), L^{2}\left(I ; \mathbf{P}^{1}\right)}+\left\langle\mathbf{A} \dot{\mathbf{y}}, \mathbf{p}_{\tau}\right\rangle_{L^{2}\left(I ; \mathbf{Y}^{0}\right), L^{2}\left(I ; \mathbf{P}^{1}\right)} .
$$

Copyright $@$ by SIAM. Unauthorized reproduction of this article is prohibited. 
Proof. Let $\tau \in(0, \infty)$ and let $(\dot{\mathbf{y}}, \dot{u}, \dot{\mathbf{p}})$ Bouligand derivative at $\tau$ in direction $\delta \tau$. To save notation it will be convenient to set

$$
b=\delta \tau\left(1+\frac{\varepsilon}{2}\left\|u_{\tau}\right\|_{L^{2}\left(I ; L^{2}(\omega)\right)}^{2}\right)+\tau \varepsilon\left(u_{\tau}, \dot{u}\right)_{L^{2}\left(I ; L^{2}(\omega)\right)} .
$$

We compute

$$
\begin{aligned}
\frac{d}{d \tau} & \mathcal{V}(\tau) \delta \tau=\delta \tau b+\frac{1}{\varepsilon}\left(\mathbf{y}_{\tau}(1)-\mathbf{z}, \dot{\mathbf{y}}(1)\right)_{\mathbf{Y}^{0}} \\
= & b+\left(\mathbf{p}_{\tau}(1), \dot{\mathbf{y}}(1)\right)_{\mathbf{L}^{2}(\Omega)} \\
= & b+\int_{0}^{1} \frac{d}{d t}\left(\mathbf{p}_{\tau}(t), \dot{\mathbf{y}}(t)\right)_{L^{2}(\Omega)}+\left(\mathbf{p}_{\tau}(0), \dot{\mathbf{y}}(0)\right)_{L^{2}(\Omega)} \\
= & b-\tau \int_{0}^{1}\left(\mathbf{A}^{*} \mathbf{p}_{\tau}(t), \dot{\mathbf{y}}(t)\right)_{\mathbf{L}^{2}(\Omega)}+\int_{0}^{1}\left(\mathbf{p}_{\tau}(t), \tau \mathbf{A} \dot{\mathbf{y}}(t)+\tau \mathbf{B} \dot{u}(t)+\delta \tau \mathbf{A y}_{\tau}(t)\right. \\
& \left.+\delta \tau \mathbf{B} u_{\tau}(t)\right)_{L^{2}(\Omega)} \\
= & \delta \tau\left(1+\frac{\varepsilon}{2}\left\|u_{\tau}\right\|_{L^{2}\left(I ; L^{2}(\omega)\right)}^{2}+\int_{0}^{1}\left(\mathbf{p}_{\tau}(t), \mathbf{A y}_{\tau}(t)+\mathbf{B} u_{\tau}(t)\right)_{\mathbf{L}^{2}(\Omega)}\right) \\
& +\tau\left(\varepsilon u_{\tau}+\mathbf{B}^{*} \mathbf{p}_{\tau}, \dot{u}\right)_{L^{2}\left(I ; L^{2}(\omega)\right)} .
\end{aligned}
$$

By (3.16) we have $\left(\varepsilon u_{\tau}+\mathbf{B}^{*} \mathbf{p}_{\tau}, \dot{u}\right)_{\left(I ; L^{2}(\Omega)\right)}=0$ and hence

$$
\frac{d}{d t} \mathcal{V}(\tau)=1+\frac{\varepsilon}{2}\left\|u_{\tau}\right\|_{L^{2}\left(I ; L^{2}(\omega)\right)}^{2}+\left(\mathbf{p}_{\tau}, \mathbf{A} \mathbf{y}_{\tau}+\mathbf{B} u_{\tau}\right)_{L^{2}\left(I ; \mathbf{L}^{2}(\omega)\right)} .
$$

This provides the expression for $\frac{d}{d t} \mathcal{V}(\tau)$. Continuity of $\tau \rightarrow \frac{d}{d t} \mathcal{V}(\tau)$ follows from Proposition 3.1 using the fact that $\left(\mathbf{p}_{\tau}, \mathbf{A} \mathbf{y}_{\tau}+\mathbf{B} u_{\tau}\right)_{L^{2}\left(I ; \mathbf{L}^{2}(\omega)\right)}=\left\langle\mathbf{p}_{\tau}, \mathbf{A} \mathbf{y}_{\tau}+\right.$ $\left.\mathbf{B} u_{\tau}\right\rangle_{L^{2}\left(I ; \mathbf{P}^{1}\right), L^{2}\left(I ; \mathbf{Y}^{0}\right)}$ implies the first claim.

Using it, we obtain for the second-order directional derivative

$$
\begin{aligned}
\left(\frac{d}{d \tau}\right)^{2} \mathcal{V}(\tau) \delta \tau= & \left(\varepsilon u_{\tau}+\mathbf{B}^{*} \mathbf{p}_{\tau}, \dot{u}\right) \\
& +\langle\mathbf{A y}+\mathbf{B} u, \dot{\mathbf{p}}\rangle_{L^{2}\left(I ; \mathbf{Y}^{0}\right), L^{2}\left(I ; \mathbf{P}^{1}\right)}+\langle\mathbf{A y}, \mathbf{p}\rangle_{L^{2}\left(I ; \mathbf{Y}^{0}\right), L^{2}\left(I ; \mathbf{P}^{1}\right)}
\end{aligned}
$$

Using once again (3.16) implies the claim.

Remark 3.9. The expression for $\frac{d}{d t} \mathcal{V}$ coincides with the left-hand side of the transversality condition. Note that due to the extra regularity requirement that $\mathbf{y}_{0} \in$ $\mathbf{Y}^{2}$ we have that $\mathbf{A y} \in L^{2}\left(I ; \mathbf{L}^{2}(\Omega)\right)$, so that we can avoid the duality pairing that we used in the transversality condition in (2.5).

We conclude this section with an asymptotic estimate of $\mathcal{V}(\tau)$ as $\tau \rightarrow \infty$.

THEOREM 3.10. Let us assume that (H1) is satisfied, which is the null-controllability of the wave equation in time $\tau_{0}>0$. Then there exist constants $\varepsilon_{0}>0$ and $c>0$ independent of $\tau$ such that for all $\tau>\tau_{0}$ and $\varepsilon \in\left(0, \varepsilon_{0}\right)$ we have

$$
\begin{aligned}
|\mathcal{V}(\tau)-\tau| & \leq c \epsilon\left(\tau-\tau_{0}\right)^{-1}, \\
\left|\frac{d}{d \tau} \mathcal{V}(\tau)-1\right| & \leq c\left(\tau-\tau_{0}\right)^{-1 / 2} .
\end{aligned}
$$

Copyright $@$ by SIAM. Unauthorized reproduction of this article is prohibited. 
Proof. Let $\sigma>0$ and a positive integer $N$ be given. From [19, Prop. 2.3] it follows that if

$$
N \geq 2 c\left(\tau_{0}\right) \sigma^{-1} \max \left(\left\|\mathbf{y}_{0}\right\|_{\mathbf{Y}^{1}},\|\mathbf{z}\|_{\mathbf{Y}^{1}}\right)
$$

holds there exists controls $u_{N, 0}$ and $u_{N, \mathbf{z}}$ with the property $\left\|u_{N, 0}\right\|_{L^{\infty}\left(I ; L^{2}(\omega)\right)}$, $\left\|u_{N, \mathbf{z}}\right\|_{L^{\infty}\left(I ; L^{2}(\omega)\right)} \leq \sigma / 2$ that drive the wave equation from $\mathbf{y}_{0}$ to 0 and from 0 to $\mathbf{z}$ in time $N \tau_{0}$, respectively. Now let $\tau>\tau_{0}$ be given, and define the positive integer $N$ such that $\tau \in\left[N \tau_{0},(N+1) \tau_{0}\right)$. Set $\sigma:=2 c\left(\tau_{0}\right) \max \left(\left\|\mathbf{y}_{0}\right\|_{\mathbf{Y}^{1}},\|\mathbf{z}\|_{\mathbf{Y}^{1}}\right) N^{-1}$; hence (3.18) holds. Then by linearity of the wave equation the controls $u_{N, 0}$ and $u_{N, \mathbf{z}}$ can be used to construct a control $u_{\tau, 0, \mathbf{z}}$ that drives the system from 0 to $\mathbf{z}$ in time $\tau$. Moreover, it holds that

$$
\left\|u_{\tau, 0, \mathbf{z}}\right\| \leq \sigma=C N^{-1} \leq C\left(\tau-\tau_{0}\right)^{-1}
$$

with a constant $C$ independent of $\tau$. Then for $\tau$ sufficiently large, $u_{\tau, 0, \mathbf{z}}$ is admissible for $\left(P_{\varepsilon}^{\tau}\right)$ and hence

$$
\tau \frac{\varepsilon}{2}\left\|u_{\tau}\right\|_{L^{2}\left(I ; L^{2}(\omega)\right)}^{2}+\frac{1}{2 \varepsilon}\left\|\mathbf{y}_{\tau}(1)-\mathbf{z}\right\|_{\mathbf{Y}^{0}}^{2} \leq \tau \frac{\varepsilon}{2}\left\|u_{\tau, 0, \mathbf{z}}\right\|_{L^{2}\left(I ; L^{2}(\omega)\right)}^{2} \leq c \varepsilon\left(\tau-\tau_{0}\right)^{-1} .
$$

This proves the first claim.

This estimate further implies that

$$
\left\|\mathbf{p}_{\tau}(1)\right\|_{\mathbf{P}^{1}}=\frac{1}{\epsilon}\left\|\mathbf{y}_{\tau}(1)-\mathbf{z}\right\|_{\mathbf{Y}^{0}} \leq c\left(\tau-\tau_{0}\right)^{-1 / 2},
$$

where $c$ is used as a generic constant, independent of $\tau$.

Testing the adjoint equations $-\mathbf{p}_{\tau, 1}^{\prime}=\tau \Delta \mathbf{p}_{\tau, 2}$ and $-\mathbf{p}_{\tau, 2}=\tau \mathbf{p}_{\tau, 1}$ by $\mathbf{p}_{\tau, 2}^{\prime}$ and $-\mathbf{p}_{\tau, 1}^{\prime}$, respectively, subtracting the resulting equations, and integrating on $(t, 1)$, $t \in(0,1)$, yields the energy equation

$$
\left\|\mathbf{p}_{\tau, 1}(t)\right\|_{L^{2}(\Omega)}^{2}+\left\|\nabla \mathbf{p}_{\tau, 2}(t)\right\|_{L^{2}(\Omega)}^{2}=\left\|\mathbf{p}_{\tau, 1}(1)\right\|_{L^{2}(\Omega)}^{2}+\left\|\nabla \mathbf{p}_{\tau, 2}(1)\right\|_{L^{2}(\Omega)}^{2}
$$

for all $t \in(0,1)$ and $\tau>0$. Together with (3.19) this proves

$$
\left\|\mathbf{p}_{\tau}\right\|_{L^{\infty}\left(I ; \mathbf{P}^{1}\right)} \leq c\left(\tau-\tau_{0}\right)^{-1 / 2} .
$$

Analogously one obtains the estimate

$$
\left\|\mathbf{y}_{\tau}\right\|_{L^{\infty}\left(\mathbf{Y}^{1}\right)} \leq c\left(\left\|\mathbf{y}_{0}\right\|_{\mathbf{Y}^{1}}+\left\|u_{\tau}\right\|_{L^{2}\left(I ; L^{2}(\omega)\right)}\right)
$$

with $c>0$ independent of $\tau$, which shows that $\mathbf{A y}_{\tau}$ is bounded in $L^{\infty}\left(I ; \mathbf{Y}^{0}\right)$ uniformly with respect to $\tau$. This implies that

$$
\sup _{t \in I}\left\langle\mathbf{A y}_{\tau}(t)+\mathbf{B} u_{\tau}(t), \mathbf{p}_{\tau}(t)\right\rangle_{\mathbf{Y}^{0}, \mathbf{P}^{1}} \leq c\left(\tau-\tau_{0}\right)^{-1 / 2},
$$

which together with Theorem 3.8 and (3.19) proves the second claim.

4. Semismooth Newton algorithm for the regularized optimality system associated to $\left(\boldsymbol{P}_{\varepsilon}^{\boldsymbol{\tau}}\right)$. The algorithm for solving $\left(P_{\varepsilon}\right)$, which will be described in the following section, relies on an efficient numerical method to solve $\left(P_{\varepsilon}^{\tau}\right)$. For this purpose we use a semismooth Newton method. In this section we verify its local superlinear convergence.

Copyright (C) by SIAM. Unauthorized reproduction of this article is prohibited. 
In view of $(3.1)$ a control $u$ is a solution to $\left(P_{\varepsilon}^{\tau}\right)$ if and only if

$$
\mathcal{F} u=u+P_{U_{a d}}\left(-\frac{1}{\varepsilon} \mathbf{B}^{*} \mathbf{p}_{\tau}\right)=0
$$

where $\mathbf{p}_{\tau}=\mathbf{p}_{\tau}(u)$ is defined through the primal and adjoint equations in (3.1). Here $\mathcal{F}$ is considered as an operator from $L^{2}\left(I ; L^{2}(\omega)\right)$ to itself. We shall verify below that $P_{U_{a d}}: C\left(\bar{I} ; L^{2}(\omega)\right) \rightarrow L^{2}\left(I ; L^{2}(\omega)\right)$ is Newton differentiable [11] with Newton derivative denoted by $D P_{U_{a d}}$. Since $u \rightarrow \mathbf{y}_{\tau} \rightarrow \mathbf{B}^{*} \mathbf{p}_{\tau}$ is a continuous linear mapping from $L^{2}\left(I ; L^{2}(\omega)\right) \rightarrow C\left(\bar{I} ; L^{2}(\omega)\right)$, it follows that $u \rightarrow \mathcal{F} u$ is Newton differentiable. To carry out the Newton iteration, given $u_{0}$, we compute $u_{k+1}=u_{k}+\delta u$ by solving

$$
D \mathcal{F}\left(u_{k}\right) \delta u=-\mathcal{F}\left(u_{k}\right),
$$

where $D \mathcal{F}(u)$ denotes the Newton derivative of $\mathcal{F}$, which is made precise below; see (4.5).

To analyze this algorithm we first address Newton differentiability of radial projections in $L^{2}(\omega)$ and $L^{2}\left(I ; L^{2}(\omega)\right)$. Differently from the Bouligand derivative, which was considered in Proposition 3.4, the Newton derivative is a bounded linear operator.

Proposition 4.1. The projection $P_{U}: L^{2}(\omega) \rightarrow L^{2}(\omega)$, which is given by $P_{U}(q)=q \min \left(1, \frac{\gamma}{\|q\|_{L^{2}(\omega)}}\right)$, is Newton differentiable with Newton derivative given by

$$
D P_{U}(q) h= \begin{cases}h & \text { if }\|q\|_{L^{2}(\omega)} \leq \gamma \\ \frac{\gamma h}{\|q\|_{L^{2}(\omega)}}-\frac{\gamma q(q, h)_{L^{2}(\omega)}}{\|q\|_{L^{2}(\omega)}^{3}} & \text { if }\|q\|_{L^{2}(\omega)}>\gamma\end{cases}
$$

Proof. The proof can be found in the appendix, section 7 .

For our purpose the projection operates pointwise in time on elements $q \in C(\bar{I}$; $\left.L^{2}(\omega)\right)$. We denote it by the same symbol.

Proposition 4.2. The projection $P_{U_{a d}}: C\left(\bar{I} ; L^{2}(\omega)\right) \rightarrow L^{2}\left(I ; L^{2}(\omega)\right)$ given by $P_{U_{a d}}(q)(t)=q(t) \min \left(1, \frac{\gamma}{\|q(t)\|_{L^{2}(\omega)}}\right)$ is Newton differentiable with Newton derivative given by $\left(D P_{U}\right) q(t)$ as in $(4.3)$.

Proof. The proof can be found in the appendix, section 7 .

Let us reconsider (4.2) and set $\mathbf{p}_{k}=\mathbf{p}\left(u_{k}\right)$, the solution to the first two equations in (3.1) with $u_{\tau}$ replaced by $u_{k}$. Let us further note that the Fréchet derivative of $u \rightarrow \mathbf{p}(u)$ at $u_{k}$ in direction $\delta_{u}$, denoted by $\mathbf{p}^{\prime}=\mathbf{p}^{\prime}\left(u_{k}\right) \delta_{u}$, satisfies

$$
\begin{cases}\partial_{t} \mathbf{y}^{\prime}=\tau \mathbf{A} \mathbf{y}^{\prime}+\tau \mathbf{B} \delta u, & \mathbf{y}^{\prime}(0)=0 \\
-\partial_{t} \mathbf{p}^{\prime}=\tau \mathbf{A}^{*} \mathbf{p}^{\prime}, & \mathbf{p}^{\prime}(1)=\frac{1}{\varepsilon}\left(\begin{array}{c}
\mathbf{y}_{1}^{\prime}(1) \\
(-\Delta)^{-1} \mathbf{y}_{2}^{\prime}(1)
\end{array}\right) .\end{cases}
$$

We further set

$$
\mathcal{I}=\left\{t \in I:\left\|\mathbf{B}^{*} \mathbf{p}_{k}(t)\right\|_{L^{2}(\omega)} \leq \varepsilon \gamma\right\} \text { and } \mathcal{A}=\left\{t \in I:\left\|\mathbf{B}^{*} \mathbf{p}_{k}(t)\right\|_{L^{2}(\omega)}>\varepsilon \gamma\right\} .
$$

Then (4.2) can equivalently be expressed as

$$
\begin{aligned}
\delta u+\frac{\gamma \chi_{\mathcal{A}}}{\left\|\mathbf{B}^{*} \mathbf{p}_{k}\right\|}\left(\mathbf{B}^{*} \mathbf{p}^{\prime}-\frac{\mathbf{B}^{*} \mathbf{p}_{k}}{\left\|\mathbf{B}^{*} \mathbf{p}_{k}\right\|}\left(\frac{\mathbf{B}^{*} \mathbf{p}_{k}}{\left\|\mathbf{B}^{*} \mathbf{p}_{k}\right\|}, \mathbf{B}^{*} \mathbf{p}^{\prime}\right)_{L^{2}(\omega)}\right)+\frac{1}{\varepsilon} \chi_{\mathcal{I}} \mathbf{B}^{*} \mathbf{p}^{\prime} \\
=-u_{k}-P_{U_{a d}}\left(\frac{1}{\varepsilon} \mathbf{B}^{*} \mathbf{p}_{k}\right)
\end{aligned}
$$

Copyright $@$ by SIAM. Unauthorized reproduction of this article is prohibited. 
where the norms are taken in $L^{2}(\omega)$. Setting $u_{k+1}=u_{k}+\delta u$ the Newton update satisfies

$$
\begin{aligned}
u_{k+1} & +\frac{1}{\varepsilon} \chi_{\mathcal{I}} \mathbf{B}^{*} \mathbf{p}_{k+1} \\
& +\frac{\gamma \chi_{\mathcal{A}}}{\left\|\mathbf{B}^{*} \mathbf{p}_{k}\right\|}\left(\mathbf{B}^{*} \mathbf{p}_{k+1}-\frac{\mathbf{B}^{*} \mathbf{p}_{k}}{\left\|\mathbf{B}^{*} \mathbf{p}_{k}\right\|}\left(\frac{\mathbf{B}^{*} \mathbf{p}_{k}}{\left\|\mathbf{B}^{*} \mathbf{p}_{k}\right\|}, \mathbf{B}^{*}\left(\mathbf{p}_{k+1}-\mathbf{p}_{k}\right)\right)_{L^{2}(\omega)}\right)=0 .
\end{aligned}
$$

In order to prove solvability of this equation, we observe that it is the necessary and sufficient optimality condition of a linear-quadratic optimal control problem.

Proposition 4.3. The Newton update $u_{k+1}$ is the unique solution to

$$
\left\{\begin{array}{l}
\min _{u \in U_{a d}} \tilde{J}(\mathbf{y}, u)=\frac{\tau}{2} \int_{0}^{1} \max \left(\frac{\left\|\mathbf{B}^{*} \mathbf{p}_{k}(t)\right\|_{L^{2}(\omega)}}{\gamma}, \varepsilon\right)\|u(t)\|^{2} d t+\frac{1}{2 \varepsilon}\|\mathbf{y}(1)-\mathbf{z}\|_{\mathbf{Y}^{0}}^{2} \\
\text { subject to } \\
\mathbf{y}_{t}=\tau \mathbf{A} \mathbf{y}+\tau \mathbf{B} u \text { on }(0,1], \quad \mathbf{y}(0)=\mathbf{y}_{0}, \\
\left(u(t), \mathbf{B}^{*} \mathbf{p}_{k}(t)\right)_{L^{2}(\omega)}=-\gamma\left\|\mathbf{B}^{*} \mathbf{p}_{k}(t)\right\|_{L^{2}(\omega)} \text { for a.e. } t \in \mathcal{A} .
\end{array}\right.
$$

Proof. Existence of a unique solution to (4.7) follows from the linear-quadratic structure of the problem. To derive the necessary and sufficient optimality system for (4.7) we consider the Lagrangian associated to (4.7), which is given by

$$
\begin{aligned}
\mathcal{L}(\mathbf{y}, u, \mathbf{p}, \mu)= & \tilde{J}(\mathbf{y}, u)+\left\langle\mathbf{p},-\mathbf{y}_{t}+\tau \mathbf{A} \mathbf{y}+\tau \mathbf{B} u\right\rangle \\
& +\left(\mu(\cdot),\left(u(\cdot), \mathbf{B}^{*} \mathbf{p}_{k}(\cdot)\right)_{L^{2}(\omega)}+\gamma\left\|\mathbf{B}^{*} \mathbf{p}_{k}(\cdot)\right\|_{L^{2}(\omega)}\right)_{L^{2}(\mathcal{A}, \mathbb{R})} .
\end{aligned}
$$

It follows that the adjoint equation for (4.7) is given by

$$
-\partial_{t} \mathbf{p}=\tau \mathbf{A}^{*} \mathbf{p}, \quad \mathbf{p}(1)=\frac{1}{\varepsilon}\left(\begin{array}{c}
\mathbf{y}_{1}(1)-z_{1} \\
(-\Delta)^{-1}\left(\mathbf{y}_{2}(1)-z_{2}\right)
\end{array}\right),
$$

and that

$$
\tau \max \left(\frac{\left\|\mathbf{B}^{*} \mathbf{p}_{k}(t)\right\|_{L^{2}(\omega)}}{\gamma}, \varepsilon\right) u+\tau \mathbf{B}^{*} \mathbf{p}+\chi_{\mathcal{A}} \mu \mathbf{B}^{*} \mathbf{p}_{k}=0 \text { a.e. on }(0,1) .
$$

Consequently

$$
u+\frac{1}{\varepsilon} \mathbf{B}^{*} \mathbf{p}=0 \text { a.e. on } \mathcal{I}
$$

and

$$
\frac{\left\|\mathbf{B}^{*} \mathbf{p}_{k}\right\|}{\gamma} u+\mathbf{B}^{*} \mathbf{p}+\frac{1}{\tau} \mu \mathbf{B}^{*} \mathbf{p}_{k}=0 \text { a.e. on } \mathcal{A} .
$$

The latter equation implies that a.e. on $\mathcal{A}$

$$
\frac{\left\|\mathbf{B}^{*} \mathbf{p}_{k}\right\|_{L^{2}(\omega)}}{\gamma}\left(u, \mathbf{B}^{*} \mathbf{p}_{k}\right)_{L^{2}(\omega)}+\left(\mathbf{B}^{*} \mathbf{p}, \mathbf{B}^{*} \mathbf{p}_{k}\right)_{L^{2}(\omega)}+\frac{1}{\tau} \mu\left\|\mathbf{B}^{*} \mathbf{p}_{k}\right\|_{L^{2}(\omega)}^{2}=0 .
$$

By the last equality in (4.7)

$$
-\left\|\mathbf{B}^{*} \mathbf{p}_{k}\right\|_{L^{2}(\omega)}^{2}+\left(\mathbf{B}^{*} \mathbf{p}, \mathbf{B}^{*} \mathbf{p}_{k}\right)_{L^{2}(\omega)}+\frac{1}{\tau} \mu\left\|\mathbf{B}^{*} \mathbf{p}_{k}\right\|_{L^{2}(\omega)}^{2}=0
$$

Copyright $@$ by SIAM. Unauthorized reproduction of this article is prohibited. 
and hence

$$
\mu=\tau-\frac{\tau}{\left\|\mathbf{B}^{*} \mathbf{p}_{k}\right\|_{L^{2}(\omega)}^{2}}\left(\mathbf{B}^{*} \mathbf{p}, \mathbf{B}^{*} \mathbf{p}_{k}\right)_{L^{2}(\omega)} \text { a.e. on } \mathcal{A} \text {. }
$$

By (4.9) we find a.e. on $\mathcal{A}$

$$
u+\frac{\gamma}{\left\|\mathbf{B}^{*} \mathbf{p}_{k}\right\|_{L^{2}(\omega)}}\left(\mathbf{B}^{*} \mathbf{p}+\mathbf{B}^{*} \mathbf{p}_{k}\left(1-\frac{\left(\mathbf{B}^{*} \mathbf{p}, \mathbf{B}^{*} \mathbf{p}_{k}\right)_{L^{2}(\omega)}}{\left\|\mathbf{B}^{*} \mathbf{p}_{k}\right\|_{L^{2}(\omega)}^{2}}\right)\right)=0
$$

or equivalently

$$
u+\frac{\gamma}{\left\|\mathbf{B}^{*} \mathbf{p}_{k}\right\|_{L^{2}(\omega)}}\left(\mathbf{B}^{*} \mathbf{p}-\frac{\mathbf{B}^{*} \mathbf{p}_{k}}{\left\|\mathbf{B}^{*} \mathbf{p}_{k}\right\|_{L^{2}(\omega)}}\left(\mathbf{B}^{*} \mathbf{p}-\mathbf{B}^{*} \mathbf{p}_{k}, \frac{\mathbf{B}^{*} \mathbf{p}_{k}}{\left\|\mathbf{B}^{*} \mathbf{p}_{k}\right\|_{L^{2}(\omega)}}\right)_{L^{2}(\omega)}\right)
$$

a.e. on $\mathcal{A}$. Combined, (4.8) and (4.10) imply that (4.6) is satisfied with $\left(u_{k+1}, \mathbf{p}_{k+1}\right)=$ $(u, \mathbf{p})$.

Uniform boundedness of the inverses of the generalized derivatives of $F$ is addressed next.

Proposition 4.4. For every $u \in L^{2}\left(I ; L^{2}(\omega)\right)$

$$
\left\|D \mathcal{F}(u)^{-1}\right\|_{\mathcal{L}\left(L^{2}\left(I ; L^{2}(\omega)\right)\right)} \leq C(u)
$$

where $C(u)>0$ is bounded on bounded subsets of $L^{2}\left(I ; L^{2}(\omega)\right)$.

Proof. Let $u, b \in L^{2}\left(I ; L^{2}(\omega)\right)$ be given. By Proposition 4.3, there exists a uniquely determined $h \in L^{2}\left(I ; L^{2}(\omega)\right)$ with $D \mathcal{F}(u) h=b$. Let $\mathbf{p}=\mathbf{p}(u)$ denote the solution to the adjoint equation as in $\left(P_{\varepsilon}^{\tau}\right)$ with $u_{\tau}$ replaced by $u$ and let $\mathbf{p}^{\prime}=\mathbf{p}^{\prime}(h)$ denote the linearization of $u \rightarrow \mathbf{p}(u)$ at $u$ in direction $h \in L^{2}\left(I ; L^{2}(\omega)\right)$, i.e., $\mathbf{p}^{\prime}$ satisfies (4.4) with $\delta u=h$.

We set

$$
\mathcal{A}=\left\{t \in I:\left\|\mathbf{B}^{*} \mathbf{p}(t)\right\|_{L^{2}(\omega)}>\varepsilon \gamma\right\}, \quad \mathcal{I}=\left\{t \in I:\left\|\mathbf{B}^{*} \mathbf{p}(t)\right\|_{L^{2}(\omega)} \leq \varepsilon \gamma\right\},
$$

and we define

$$
q=\frac{\mathbf{B}^{*} \mathbf{p}}{\left\|\mathbf{B}^{*} \mathbf{p}\right\|_{L^{2}(\omega)}} \chi_{\mathcal{A}}
$$

We have the orthogonal decomposition

$$
\begin{aligned}
h & =\chi_{\mathcal{I}} h+\chi_{\mathcal{A}}\left(h-(h, q)_{L^{2}(\omega)} q\right)+\chi_{\mathcal{A}}(h, q)_{L^{2}(\omega)} q \\
& =h_{\mathcal{I}}+h_{T}+h_{N}
\end{aligned}
$$

of $h$ into inactive, tangential, and normal components. Likewise, we split $b=b_{\mathcal{I}}+b_{N}+$ $b_{T}$ and $\mathbf{B}^{*} \mathbf{p}^{\prime}(h)=\left(\mathbf{B}^{*} \mathbf{p}^{\prime}(h)\right)_{\mathcal{I}}+\left(\mathbf{B}^{*} \mathbf{p}^{\prime}(h)\right)_{T}+\left(\mathbf{B}^{*} \mathbf{p}^{\prime}(h)\right)_{N}$. The equation $D \mathcal{F}(u) h=b$ implies, see (4.6), that these components satisfy the system

$$
\begin{aligned}
h_{\mathcal{I}}+\frac{1}{\varepsilon}\left(\mathbf{B}^{*} \mathbf{p}^{\prime}(h)\right)_{\mathcal{I}} & =b_{\mathcal{I}}, \\
h_{T}+\frac{\gamma}{\left\|\mathbf{B}^{*} \mathbf{p}\right\|_{L^{2}(\omega)}}\left(\mathbf{B}^{*} \mathbf{p}^{\prime}(h)\right)_{T} & =b_{T}, \\
h_{N} & =b_{N} .
\end{aligned}
$$

Copyright $@$ by SIAM. Unauthorized reproduction of this article is prohibited. 
Hence, we have $\left\|h_{N}\right\|_{L^{2}\left(I ; L^{2}(\omega)\right)}=\left\|b_{N}\right\|_{L^{2}\left(I ; L^{2}(\omega)\right)}$. Moreover, by testing the first two equations with $h$, integrating on $I$, and adding them, we obtain

$$
\begin{aligned}
& \varepsilon\left\|h_{\mathcal{I}}\right\|_{L^{2}\left(I ; L^{2}(\omega)\right)}^{2}+\int_{\mathcal{A}} \frac{\left\|\mathbf{B}^{*} \mathbf{p}\right\|_{L^{2}(\omega)}}{\gamma}\left\|h_{T}\right\|_{\left.L^{2}(\omega)\right)}^{2}+\left(\mathbf{B}^{*} \mathbf{p}^{\prime}(h), h\right)_{L^{2}\left(I ; L^{2}(\omega)\right)} \\
& =\left(\mathbf{B}^{*} \mathbf{p}^{\prime}(h), h_{N}\right)_{L^{2}\left(I ; L^{2}(\omega)\right)}+\varepsilon\left(b_{I}, h_{I}\right)+\int_{\mathcal{A}} \frac{\left\|\mathbf{B}^{*} \mathbf{p}\right\|_{L^{2}(\omega)}}{\gamma}\left(b_{T}, h_{T}\right)_{\left.L^{2}(\omega)\right)} .
\end{aligned}
$$

Since $\left\|\mathbf{B}^{*} \mathbf{p}(t)\right\|_{L^{2}(\omega)}>\varepsilon \gamma$ on $\mathcal{A}$ and $h_{N}=b_{N}$, we can estimate using Young's inequality

$$
\begin{aligned}
\frac{\varepsilon}{2}\left\|h_{\mathcal{I}}+h_{T}\right\|_{L^{2}\left(I ; L^{2}(\omega)\right)}^{2}+\left(\mathbf{B}^{*} \mathbf{p}^{\prime}(h), h\right)_{L^{2}\left(I ; L^{2}(\omega)\right)} \\
\leq \\
\quad\left(\mathbf{B}^{*} \mathbf{p}^{\prime}(h), h_{N}\right)_{L^{2}\left(I ; L^{2}(\omega)\right)} \\
\quad+\frac{1}{2} \max \left(\varepsilon, \frac{1}{\gamma}\left\|\mathbf{B}^{*} \mathbf{p}\right\|_{L^{\infty}\left(I ; L^{2}(\omega)\right)}\right)\left\|b_{I}+b_{N}\right\|_{L^{2}\left(I ; L^{2}(\omega)\right)}^{2}
\end{aligned}
$$

We find

$$
\begin{aligned}
\tau\left(\mathbf{B}^{*} \mathbf{p}^{\prime}(h), h\right)_{L^{2}\left(I ; L^{2}(\omega)\right)} & =\left\langle\mathbf{p}^{\prime}(h), \partial_{t} \mathbf{y}^{\prime}-\tau \mathbf{A} \mathbf{y}^{\prime}\right\rangle=\left(\mathbf{p}^{\prime}(h)(1), \mathbf{y}^{\prime}(1)\right) \\
& =\frac{1}{\varepsilon}\left\|\mathbf{y}^{\prime}(1)\right\|_{\mathbf{Y}^{0}}^{2}=\varepsilon\left\|\mathbf{p}^{\prime}(1)\right\|_{\mathbf{P}^{1}}^{2},
\end{aligned}
$$

where $\mathbf{y}^{\prime}$ is the solution of the first equation in (4.4) with $\delta u$ replaced by $h$. By the properties of the wave equation, we obtain

$$
\left\|\mathbf{B}^{*} \mathbf{p}^{\prime}(h)\right\|_{L^{\infty}\left(I ; L^{2}(\omega)\right)} \leq\left\|\mathbf{p}^{\prime}\right\|_{C\left(\bar{I}, \mathbf{P}^{0}\right)} \leq c\left\|\mathbf{p}^{\prime}\right\|_{C\left(\bar{I}, \mathbf{P}^{1}\right)} \leq c\left\|\mathbf{p}^{\prime}(1)\right\|_{\mathbf{P}^{1}}
$$

with a constant $c>0$ independent of $u, \tau$. Using (4.12) and (4.13) in (4.11), we obtain

$$
\begin{aligned}
& \varepsilon\left\|h_{\mathcal{I}}+h_{T}\right\|_{L^{2}\left(I ; L^{2}(\omega)\right)}^{2}+\varepsilon\left\|\mathbf{p}^{\prime}(1)\right\|_{\mathbf{P}^{1}}^{2} \\
& \leq \frac{c^{2}}{\varepsilon}\left\|b_{N}\right\|_{L^{2}\left(I ; L^{2}(\omega)\right)}^{2} \\
& \quad+\max \left(\varepsilon, \frac{1}{\gamma}\left\|\mathbf{B}^{*} \mathbf{p}\right\|_{L^{\infty}\left(I ; L^{2}(\omega)\right)}\right)\left\|b_{I}+b_{N}\right\|_{L^{2}\left(I ; L^{2}(\omega)\right)}^{2}
\end{aligned}
$$

which implies the claim.

We are now prepared to verify local convergence of the semismooth Newton iteration (4.2).

THEOREM 4.5. Assume that $\left\|u_{0}-u_{\tau}\right\|_{L^{2}\left(I ; L^{2}(\omega)\right)}$ is sufficiently small.

Then the semismooth Newton iteration (4.2) converges superlinearly to $u_{\tau}$ in $L^{2}(I$; $\left.L^{2}(\omega)\right)$.

Proof. The mapping $\mathcal{F}$ is continuous and by Proposition 4.2 is also Newton differentiable at every $u \in L^{2}\left(I ; L^{2}(\omega)\right)$. By Proposition 4.4 the inverses of $D \mathcal{F}(u)$ are uniformly bounded. The conclusion therefore follows from well-known results on semismooth Newton methods; cf. [11, p. 238].

5. A semismooth Newton algorithm. In this section, we propose a nested Newton-type algorithm for solving $(\tilde{P})$. Furthermore, we will report on numerical results.

Copyright (c) by SIAM. Unauthorized reproduction of this article is prohibited. 
5.1. Description of the algorithm. The principal idea of our algorithm is to exploit the hierarchy of optimization problems presented in this article: the original problem $(\tilde{P})$, the penalized problems $\left(P_{\varepsilon}\right)$ for $\epsilon \searrow 0$, and the parametric problems $\left(P_{\varepsilon}^{\tau}\right)$ for fixed $\epsilon>0, \tau \geq 0$.

In the algorithm, the problem $(\tilde{P})$ is solved by means of solving a sequence of problems of type $\left(P_{\varepsilon}\right)$ for $\varepsilon \rightarrow 0$. Thus in the outer loop of Algorithm 1 below the regularization parameter $\epsilon$ is driven to 0 . Within each step $i$ of this loop, the problem $\left(P_{\varepsilon}\right)$ has to be solved with a positive penalization parameter $\epsilon_{i}>0$. The solution of $\left(P_{\varepsilon}\right)$ with respect to $(\tau, \mathbf{y}, u, \mathbf{p})$ is carried out as is a Newton algorithm for the minimization of the functional $\mathcal{V}$, defined in (3.17) by

$$
\mathcal{V}(\tau)=\tau\left(1+\frac{\varepsilon}{2}\left\|u_{\tau}\right\|_{L^{2}\left(I ; L^{2}(\omega)\right)}^{2}\right)+\frac{1}{2 \varepsilon}\left\|\mathbf{y}_{\tau}(1)-\mathbf{z}\right\|_{\mathbf{Y}^{0}}^{2}
$$

subject to the constraint $\tau \geq 0$. In the definition of $\mathcal{V}$, the pair $\left(\mathbf{y}_{\tau}, u_{\tau}\right)$ is the unique solution of the parametric optimization problem $\left(P_{\varepsilon}^{\tau}\right)$. The first and second derivatives of $\mathcal{V}$ are computed on the basis of Theorems 3.6 and 3.8. In order to evaluate $\mathcal{V}$ problem $\left(P_{\varepsilon}^{\tau}\right)$ has to be solved for fixed $\epsilon$ and $\tau$. Here we use the semismooth Newton method, which was analyzed in section 4 . The overall solution procedure is depicted in Algorithm 1. The detailed algorithm to solve the subproblems $\left(P_{\varepsilon}\right)$ is described in Algorithm 2.

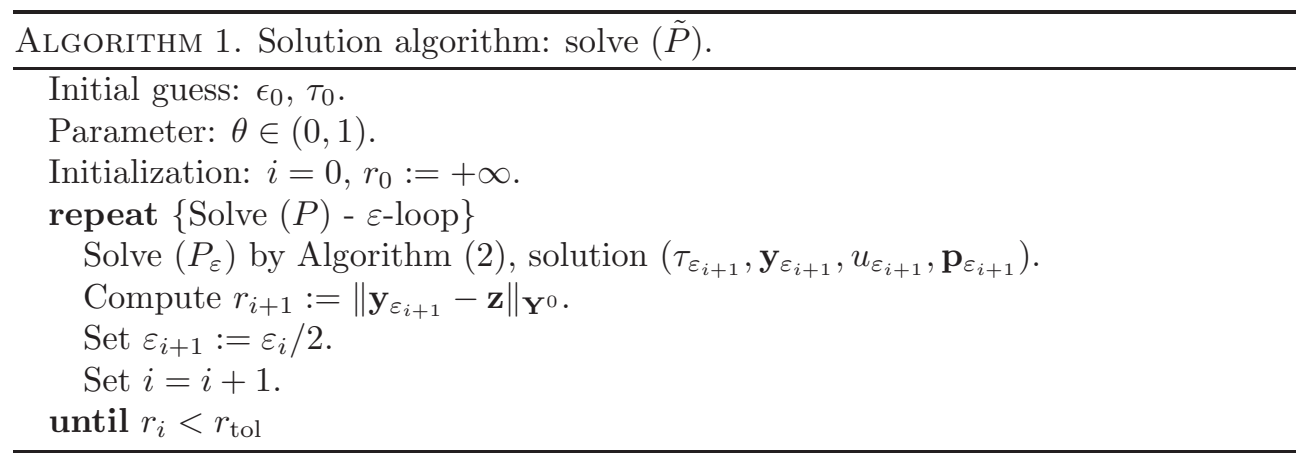

As stopping criterion for the solution of the regularized problem $\left(P_{\varepsilon_{i}}\right)$ by Algorithm 2 , we chose

$$
\left|\tau_{k}-\max \left(0, \tau_{k}-\frac{d}{d \tau} \mathcal{V}\left(\tau_{k}\right)\right)\right|<\varepsilon_{i}
$$

which is motivated by the necessary optimality condition of projected gradient type

$$
\tau_{\varepsilon}-\max \left(0, \tau_{\varepsilon}-\frac{d}{d \tau} \mathcal{V}\left(\tau_{\varepsilon}\right)\right)=0
$$

for $\left(P_{\varepsilon}\right)$. That is, the precision in which the $\left(P_{\varepsilon}\right)$ is solved is directly coupled to the regularization parameter. This prevents the overall algorithm from spending too much time to solve problems with relatively large regularization parameters.

To apply a nonsmooth Newton's method for the minimization of $\mathcal{V}$, the secondorder directional derivative of $\mathcal{V}$ is computed by solving the sensitivity system (3.10). We observed that the computational effort to solve (3.10) is comparable to the effort 


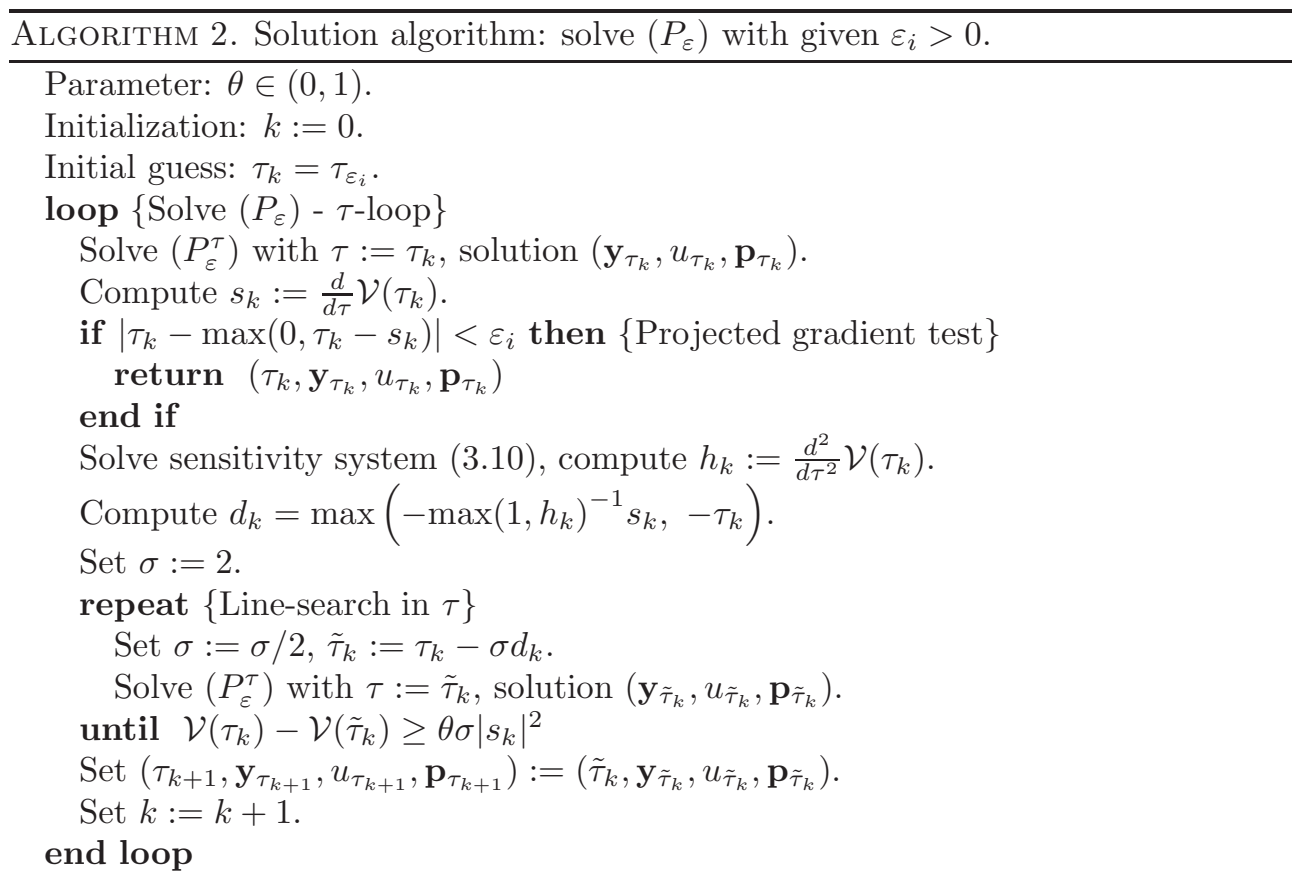

of computing one semismooth Newton step for $\left(P_{\varepsilon}^{\tau}\right)$. Once the solution of (3.10) is available, the second-order directional derivative of $\mathcal{V}$ is obtained from

$$
\left(\frac{d}{d \tau}\right)^{2} \mathcal{V}(\tau) \delta \tau=\langle\mathbf{A y}+\mathbf{B} u, \dot{\mathbf{p}}\rangle_{L^{2}\left(I ; \mathbf{Y}^{0}\right), L^{2}\left(I ; \mathbf{P}^{1}\right)}+\langle\mathbf{A} \dot{\mathbf{y}}, \mathbf{p}\rangle_{L^{2}\left(I ; \mathbf{Y}^{0}\right), L^{2}\left(I ; \mathbf{P}^{1}\right)}
$$

With the values $\frac{d}{d \tau} \mathcal{V}\left(\tau_{k}\right)$ and $\left(\frac{d}{d \tau}\right)^{2} \mathcal{V}\left(\tau_{k}\right)$ computed, an Armijo-type line-search with feasible directions is used to determine the next iterate $\tau_{k+1}$.

For the innermost semismooth Newton iteration, which solves the parametric optimization problem $\left(P_{\varepsilon}^{\tau}\right)$, an Armijo-type line-search is used. The iteration is stopped as soon as the residual at the current iterate $u_{\tau_{k}}^{j}$ satisfies

$$
\left\|\mathcal{F}\left(u_{\tau_{k}}^{j}\right)\right\|_{L^{2}\left(I ; L^{2}(\omega)\right)} \leq \max \left(10^{-2}\left|\frac{d}{d \tau} \mathcal{V}\left(\tau_{k-1}\right)\right|, 10^{-6}\right) ;
$$

see (4.1) for the definition of $\mathcal{F}$.

Let us now briefly sketch the arguments to show convergence of Algorithm 1. The convergence of solutions $\left(\tau_{\varepsilon}, \mathbf{y}_{\varepsilon}, u_{\varepsilon}\right)$ for $\varepsilon \rightarrow 0$ to solutions of $(\tilde{P})$ was analyzed in our previous work [19]. The use of the inexact stopping criterion (5.1) is justified by the numerical observation that $\left(\frac{d}{d \tau}\right)^{2} \mathcal{V}\left(\tau_{k}\right)>0$ near solutions of $\left(P_{\varepsilon}\right)$. Algorithm 2 to solve $\left(P_{\varepsilon}\right)$ is itself a globalized Newton method, which is known to be globally convergent. The convergence of the semismooth Newton method to solve $\left(P_{\varepsilon}^{\tau}\right)$ was proven in Theorem 4.5.

Turning to the discretization scheme, the state, adjoint, and control variables were discretized by finite elements. The amplitude components $\mathbf{y}_{1}$ and $\mathbf{p}_{2}$ are discretized by P1-elements, while P0-elements are used for the velocity unknowns $\mathbf{y}_{2}$ and $\mathbf{p}_{1}$ and for the controls.

For time discretization, we used a $\operatorname{cg}(1) \mathrm{dG}(0)$-scheme as described in [18], which corresponds to a Crank-Nicolson time-stepping procedure. 
5.2. Numerical experiments. Let us report on the outcome of our computational experiments. We modified the cost functional of the penalized problem $\left(P_{\varepsilon}\right)$ to

$$
J_{\varepsilon}(\tau, u)=\tau\left(1+\frac{\varepsilon}{2}\|u\|_{L^{2}\left(I ; L^{2}(\omega)\right)}^{2}\right)+\frac{1}{2 \varepsilon_{y}}\|\mathbf{y}(1)-\mathbf{z}\|_{\mathbf{Y}^{0}}^{2}
$$

with

$$
\varepsilon_{y}=0.1 \cdot \varepsilon \text {. }
$$

That is, the violation of the terminal constraint is penalized with larger weight.

We chose $\Omega=(0,1)^{2}$ for our computations. The control bound was set to $\gamma=3$. The target state was $z_{1}=z_{2}=0$ and the initial state was given as $y_{1}\left(x_{1}, x_{2}\right)=$ $x_{1} x_{2}\left(1-x_{1}\right)\left(1-x_{2}\right)$.

The spatial domain was discretized using a uniform triangulation, and the time interval was split into equidistant subintervals. We will report on the results for the following hierarchy of discretizations: $(N, M)=(50,10),(200,20),(800,40)$, and $(3200,80)$, where $N$ is the numbers of triangles and $M$ the numbers of time intervals. The resulting mesh size $h$ is $h=2 / \sqrt{N}$, the resulting length of the temporal subintervals $\Delta t=1 / M$.

The parameters for the algorithm as described in the previous section were chosen as

$$
\varepsilon_{0}=0.1, \quad \tau_{0}=1.9, \quad \theta=10^{-3} .
$$

The algorithm was stopped as soon as the terminal residual satisfies $\left\|\mathbf{y}_{\varepsilon}(1)-\mathbf{z}\right\|_{\mathbf{Y}^{0}} \leq$ $10^{-3}$, which corresponds to the choice $r_{\text {tol }}=10^{-3}$.

In the examples which follow, we also pay special attention to the behavior of the boundedness of $\left\{\left\|\mathbf{p}_{\varepsilon, h}(1)\right\|_{\mathbf{P}^{1}}\right\}$ and $\left\{\left\|\mathbf{p}_{\varepsilon, h}(1)\right\|_{\mathbf{P}^{0}}\right\}$ with respect to $\varepsilon$. By Theorem 2.5, we have that if $\left\{\left\|\mathbf{p}_{\varepsilon}(1)\right\|_{\mathbf{P}^{1}}\right\}$ is bounded, then (2.6) and

$$
1+\langle\mathbf{A z}+\mathbf{B} \tilde{u}(1), \tilde{\mathbf{p}}(1)\rangle_{\mathbf{Y}^{1}, \mathbf{P}^{0}}=0
$$

are satisfied for some adjoint state $\tilde{\mathbf{p}}$. In the case that $\left\{\left\|\mathbf{p}_{\varepsilon}(1)\right\|_{\mathbf{P}^{0}}\right\}$ is unbounded, there is $\tilde{\mathbf{p}} \neq 0$ satisfying (2.6) and

$$
\langle\mathbf{A z}+\mathbf{B} \tilde{u}(1), \tilde{\mathbf{p}}(1)\rangle_{\mathbf{Y}^{1}, \mathbf{P}^{0}}=0 .
$$

It is still an open question to further analyze under which assumptions on the data of the control problem $(\tilde{P})$ we can expect the transversality condition to be in qualified (5.2) or unqualified form (5.3). For the details we refer to [19].

Let us briefly comment on our experience with solving problem $\left(P_{\varepsilon}\right)$ directly for $(\tau, \mathbf{y}, u, \mathbf{p})$ by the semismooth Newton method. This requires applying the semismooth Newton method to iteratively solve the optimality system (2.5). It turned out that the bilevel optimization analyzed here was clearly more robust for the problems that we tested. For this reason we gave preference to it in the present work.

Example 1. Here the initial velocity was $y_{2}=0$. That is, the control objective was to steer the system from a given deflection into zero. The control domain was chosen to be $\omega:=\Omega$.

The most delicate issue is the behavior of the algorithm with respect to the asymptotic behavior $\varepsilon \rightarrow 0^{+}$. So let us report on it first. 

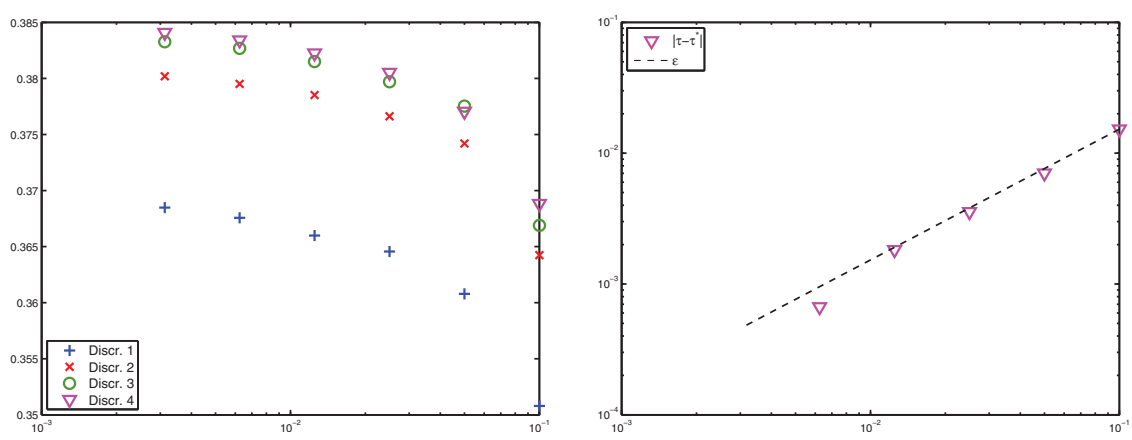

Fig. 1. Example 1. $\tau_{\varepsilon}$ versus $\varepsilon$ for different discretizations; $\left|\tau_{\varepsilon}-\tau^{*}\right|$ versus $\varepsilon$ for finest discretization.

TABLE 1

Example 1. Convergence history.

\begin{tabular}{ccrr}
\hline$\varepsilon$ & $\tau_{\varepsilon}$ & $\left|\tau_{\varepsilon}-\tau^{*}\right|$ & $\left\|\mathbf{y}_{\varepsilon}(1)-\mathbf{z}\right\|_{\mathbf{Y}^{0}}$ \\
\hline $1.0000 \cdot 10^{-1}$ & $3.68 \cdot 10^{-1}$ & $1.52 \cdot 10^{-2}$ & $2.49 \cdot 10^{-2}$ \\
$5.0000 \cdot 10^{-2}$ & $3.77 \cdot 10^{-1}$ & $7.03 \cdot 10^{-3}$ & $1.03 \cdot 10^{-2}$ \\
$2.5000 \cdot 10^{-2}$ & $3.80 \cdot 10^{-1}$ & $3.56 \cdot 10^{-3}$ & $4.48 \cdot 10^{-3}$ \\
$1.2500 \cdot 10^{-2}$ & $3.82 \cdot 10^{-1}$ & $1.82 \cdot 10^{-3}$ & $2.12 \cdot 10^{-3}$ \\
$6.2500 \cdot 10^{-3}$ & $3.83 \cdot 10^{-1}$ & $6.67 \cdot 10^{-4}$ & $1.02 \cdot 10^{-3}$ \\
$3.1250 \cdot 10^{-3}$ & $3.84 \cdot 10^{-1}$ & & $5.10 \cdot 10^{-4}$ \\
\hline
\end{tabular}

In Figure 1 we show the convergence of $\tau_{\varepsilon}$ for different discretizations. Moreover, we depict the evolution of $\left|\tau_{\varepsilon}-\tau^{*}\right|$ for the finest discretization, where we use as value for $\tau^{*}$ the optimal time for the smallest $\varepsilon_{i}$, i.e., $\tau^{*}=\tau_{\varepsilon_{i_{\min }}}$. In Table 1 we report on the convergence of $\tau_{\varepsilon}$ and $\left\|\mathbf{y}_{\varepsilon}(1)-\mathbf{z}\right\|_{\mathbf{Y}^{0}}$ for the finest discretization. We observe the convergence rate

$$
\left|\tau_{\varepsilon}-\tau^{*}\right|=O(\varepsilon) .
$$

As argued in [19, Corollary 3.3] this implies that

$$
\left\|\mathbf{y}_{\varepsilon}(1)-\mathbf{z}\right\|_{\mathbf{Y}^{0}}=O(\varepsilon),
$$

which can be seen in Table 1 as well. Moreover, since by (2.5) we have $\left\|\mathbf{p}_{\varepsilon}(1)\right\|_{\mathbf{P}^{1}}=$ $\frac{1}{\varepsilon}\left\|\mathbf{y}_{\varepsilon}(1)-\mathbf{z}\right\|_{\mathbf{Y}^{0}}$ this convergence rate implies that $\left\{\mathbf{p}_{\varepsilon}(1)\right\}$ is bounded in $\mathbf{P}^{1}$. To address the effect of discretization we plotted in Figure 2 the evolution of $\left\|\mathbf{p}_{\varepsilon, h}(1)\right\|_{\mathbf{P}^{1}}$ for the solutions of the discrete problems for the four different discretizations, and we observed that the $\mathbf{P}^{1}$-norms of $\mathbf{p}_{\varepsilon, h}(1)$ are bounded uniformly with respect to $\varepsilon$ and with respect to the discretization. This suggests that the continuous norms $\left\{\left\|\mathbf{p}_{\varepsilon}(1)\right\|_{\mathbf{P}^{1}}\right\}$ are bounded as well. Hence, we expect that the limits $\left(\tau^{*}, \tilde{\mathbf{y}}, \tilde{u}, \tilde{\mathbf{p}}\right)$ of solutions $\left(\tau_{\epsilon}, \mathbf{y}_{\epsilon}, u_{\epsilon}, \mathbf{p}_{\epsilon}\right)$ for $\epsilon \rightarrow 0$ satisfy the transversality condition in the qualified form (5.2).

Table 2 depicts the behavior of the number of iterations of the middle and the innermost loops of Algorithm 1, i.e., the $\tau$ and the semismooth Newton loops, as a function of a decreasing sequence of $\varepsilon_{i}$ values. Except for the solution for the initial $\varepsilon$ value, the number of $\tau$ iterations increases as $\varepsilon_{i}$ decreases. The number of semismooth Newton iterations necessary for each fixed value of $\varepsilon$ and $\tau$ remains almost constant for the example shown here. 


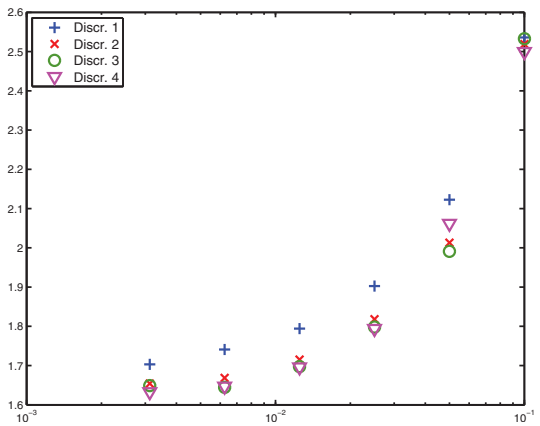

Fig. 2. Example 1. $\left\|\mathbf{p}_{\varepsilon}(1)\right\|_{\mathbf{P}^{1}}$ versus $\varepsilon$ for different discretizations.

TABLE 2

Example 1. Iteration numbers.

\begin{tabular}{lrr}
\hline$\varepsilon$ & $\tau$-iterations & ssn-iterations \\
\hline $1.0000 \cdot 10^{-1}$ & 9 & 31 \\
$5.0000 \cdot 10^{-2}$ & 1 & 10 \\
$2.5000 \cdot 10^{-2}$ & 2 & 17 \\
$1.2500 \cdot 10^{-2}$ & 25 & 241 \\
$6.2500 \cdot 10^{-3}$ & 20 & 266 \\
$3.1250 \cdot 10^{-3}$ & 30 & 470 \\
\hline
\end{tabular}

TABLE 3

Example 2. Convergence history.

\begin{tabular}{ccrr}
\hline$\varepsilon$ & $\tau_{\varepsilon}$ & $\left|\tau_{\varepsilon}-\tau^{*}\right|$ & $\left\|\mathbf{y}_{\varepsilon}(1)-\mathbf{z}\right\|_{\mathbf{Y}^{0}}$ \\
\hline $1.0000 \cdot 10^{-1}$ & $4.00 \cdot 10^{-1}$ & $3.15 \cdot 10^{-2}$ & $2.87 \cdot 10^{-2}$ \\
$5.0000 \cdot 10^{-2}$ & $4.18 \cdot 10^{-1}$ & $1.45 \cdot 10^{-2}$ & $1.21 \cdot 10^{-2}$ \\
$2.5000 \cdot 10^{-2}$ & $4.24 \cdot 10^{-1}$ & $7.81 \cdot 10^{-3}$ & $5.68 \cdot 10^{-3}$ \\
$1.2500 \cdot 10^{-2}$ & $4.28 \cdot 10^{-1}$ & $3.72 \cdot 10^{-3}$ & $2.71 \cdot 10^{-3}$ \\
$6.2500 \cdot 10^{-3}$ & $4.31 \cdot 10^{-1}$ & $1.34 \cdot 10^{-3}$ & $1.33 \cdot 10^{-3}$ \\
$3.1250 \cdot 10^{-3}$ & $4.32 \cdot 10^{-1}$ & & $7.00 \cdot 10^{-4}$ \\
\hline
\end{tabular}

Example 2. In our second example we chose the initial velocity to be $y_{2}\left(x_{1}, x_{2}\right)=$ $x_{1}^{2}+y_{2}^{2}$. All other data are the same as in the previous example. The convergence history can be found in Table 3. We observed the same convergence rates as in the previous example, i.e., $\left\|\mathbf{y}_{\varepsilon}(1)-\mathbf{z}\right\|_{\mathbf{Y}^{0}}=O(\varepsilon)$. Moreover, $\left\{\mathbf{p}_{\varepsilon}(1)\right\}$ is bounded in $\mathbf{P}^{1}$, which indicates that the qualified transversality condition (5.2) holds for the continuous original problem.

Example 3. Here again the initial velocity was $y_{2}=0$, however, the control domain $\omega$ was chosen to be a proper subset of $\Omega$ : $\omega=\Omega \backslash[0,0.5]^{2}$.

The convergence rates are worse than in Examples 1 and 2. In fact, in the computations we observe that $\left|\tau_{\varepsilon}-\tau^{*}\right|=O(\sqrt{\varepsilon})$; see Table 4 and Figure 3, which coincide with the theoretical findings [19, Cor. 3.3]. The numerical optimal time was found to be $\tau^{*} \approx 0.62$.

Moreover, the norms $\left\{\left\|\mathbf{p}_{\varepsilon, h}(1)\right\|_{\mathbf{P}^{1}}\right\}_{\varepsilon>0}$ are not bounded uniformly with respect to the discretization, see Figure 4 , and $\left\{\left\|\mathbf{p}_{\varepsilon(1), h}\right\|_{\mathbf{P}^{0}}\right\}_{\varepsilon>0}$ is not bounded either. This suggests that for the original system the transversality condition holds in the unqualified form $\langle\mathbf{B} u(1), \tilde{\mathbf{p}}(1)\rangle_{\left(\mathbf{P}^{0}\right)^{*}, \mathbf{P}^{0}}=0$, cf. (5.3) with $\mathbf{z}=0$, which was also observed numerically.

Further we observed a mesh-dependent convergence of the algorithm. For finer discretizations, smaller values of the parameter $\varepsilon$ were needed to reach the prescribed 
TABLE 4

Example 3. Convergence history.

\begin{tabular}{cccc}
\hline$\varepsilon$ & $\tau_{\varepsilon}$ & $\left|\tau_{\varepsilon}-\tau^{*}\right|$ & $\left\|\mathbf{y}_{\varepsilon}(1)-\mathbf{z}\right\|_{\mathbf{Y}^{0}}$ \\
\hline $1.0000 \cdot 10^{-1}$ & $3.98 \cdot 10^{-1}$ & $2.25 \cdot 10^{-1}$ & $3.97 \cdot 10^{-2}$ \\
$5.0000 \cdot 10^{-2}$ & $4.45 \cdot 10^{-1}$ & $1.77 \cdot 10^{-1}$ & $2.41 \cdot 10^{-2}$ \\
$2.5000 \cdot 10^{-2}$ & $4.86 \cdot 10^{-1}$ & $1.37 \cdot 10^{-1}$ & $1.50 \cdot 10^{-2}$ \\
$1.2500 \cdot 10^{-2}$ & $5.21 \cdot 10^{-1}$ & $1.02 \cdot 10^{-1}$ & $9.25 \cdot 10^{-3}$ \\
$6.2500 \cdot 10^{-3}$ & $5.47 \cdot 10^{-1}$ & $7.64 \cdot 10^{-2}$ & $5.81 \cdot 10^{-3}$ \\
$3.1250 \cdot 10^{-3}$ & $5.67 \cdot 10^{-1}$ & $5.58 \cdot 10^{-2}$ & $3.74 \cdot 10^{-3}$ \\
$1.5625 \cdot 10^{-3}$ & $5.84 \cdot 10^{-1}$ & $3.95 \cdot 10^{-2}$ & $2.52 \cdot 10^{-3}$ \\
$7.8125 \cdot 10^{-4}$ & $5.98 \cdot 10^{-1}$ & $2.50 \cdot 10^{-2}$ & $1.73 \cdot 10^{-3}$ \\
$3.9063 \cdot 10^{-4}$ & $6.12 \cdot 10^{-1}$ & $1.15 \cdot 10^{-2}$ & $1.21 \cdot 10^{-3}$ \\
$1.9531 \cdot 10^{-4}$ & $6.23 \cdot 10^{-1}$ & & $9.02 \cdot 10^{-4}$ \\
\hline
\end{tabular}
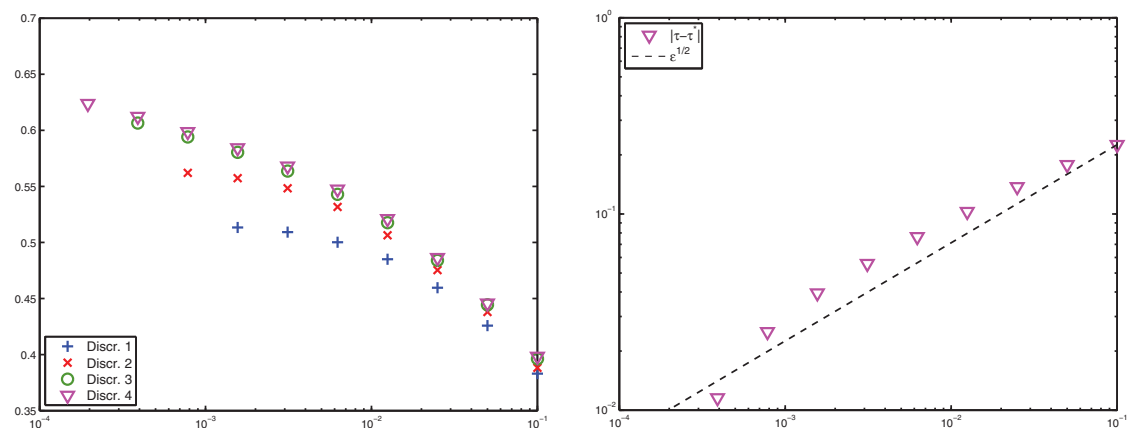

FIG. 3. Example 3. $\tau_{\varepsilon}$ versus $\varepsilon$ for different discretizations; $\left|\tau_{\varepsilon}-\tau^{*}\right|$ versus $\sqrt{\varepsilon}$ for finest discretization.
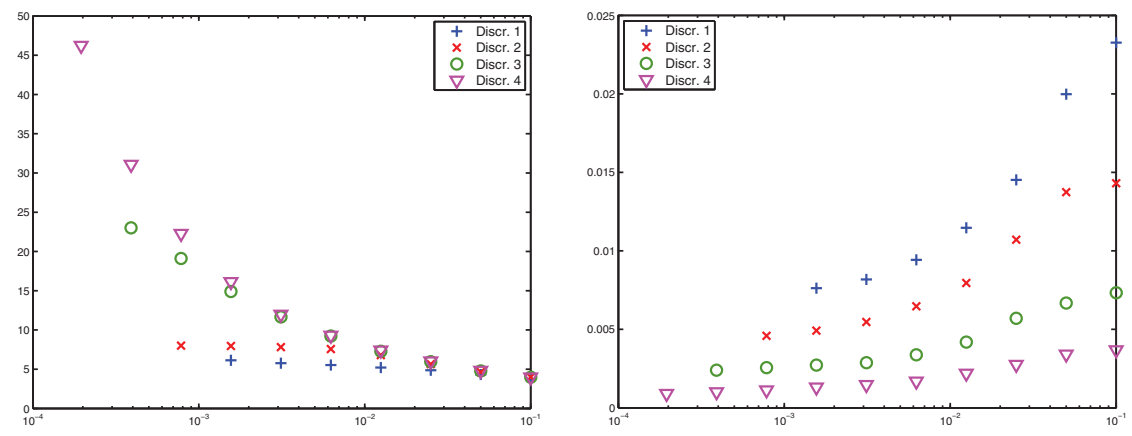

FIG. 4. Example 3. $\left\|\mathbf{p}_{\varepsilon}(1)\right\|_{\mathbf{P}^{1}}$ and $\frac{\left\|\mathbf{p}_{\varepsilon}(1)\right\|_{\mathbf{P}^{1}}}{\left\|\mathbf{p}_{\varepsilon}(1)\right\|_{\mathbf{P}^{0}}}$ versus $\varepsilon$ for different discretizations.

accuracy for the terminal residual; see Figure 3. Consequently, the algorithm needs more iterations on finer discretizations.

Finally we observe that for this control domain, controllability is guaranteed for times $T>\sqrt{2}$, as the geometrical condition of [1] is satisfied for this configuration: Each ray of geometric optics starting in $\Omega$ will hit the control domain $\omega$ within time $t=\sqrt{2}$.

6. Concluding remarks. Clearly many interesting questions can be addressed as extensions of the present research. Here we used an $L^{2}(\omega)$-norm constraint on the controls spatially. Pointwise controls are equally important and require, in part, 
different treatment. Boundary control problems, as well, are a natural problem to be addressed. Various aspects of discretization would also be of interest.

7. Appendix: Proofs. Here we present the proofs of those results which are not provided in sections 3 and 4 . Let us recall the definitions of the projections onto $U_{a d}$ and $U$ :

$$
\left(P_{U_{a d}}(u)\right)(t):=P_{U}(u(t)) \text { f.a.a. } t \in(0,1)
$$

where the projection onto $U$ is defined as

$$
P_{U}(u)=\min \left(1, \frac{\gamma}{\|u\|_{L^{2}(\omega)}}\right)
$$

In what follows, we will briefly recall the statements of the lemmas and propositions as presented earlier in sections 3 and 4 .

Lemma (Lemma 3.2). The projection $P_{U_{a d}}$ is continuous mapping from $C^{1}(\bar{I}$; $\left.L^{2}(\omega)\right)$ to $H^{1}\left(I ; L^{2}(\omega)\right)$.

Proof of Lemma 3.2. The proof follows the ideas of a related result in [15] on the continuity of the operator $y \mapsto \max (y, 0)$ in $H^{1}(\Omega)$. Let a sequence $q_{n}$ be given with $q_{n} \rightarrow q$ in $C^{1}\left(\bar{I} ; L^{2}(\omega)\right)$. We have for the time derivative

$$
\partial_{t} P_{U}(q(t))= \begin{cases}\partial_{t} q(t) & \text { if }\|q(t)\|_{L^{2}(\omega)} \leq \gamma \\ \gamma \frac{\|q(t)\|_{L^{2}(\omega)}^{2} \partial_{t} q(t)-\left(q(t), \partial_{t} q(t)\right)_{L^{2}(\omega)} q(t)}{\|q(t)\|_{L^{2}(\omega)}^{3}} & \text { if }\|q(t)\|_{L^{2}(\omega)}>\gamma\end{cases}
$$

for almost every $t \in I$. Here we use that $\left(q(t), \partial_{t} q(t)\right)=0$ for a.e. $t \in I$ such that $\|q(t)\|_{L^{2}(\omega)}=\gamma$; cf., e.g., [15, Lem. A.4].

It follows that $\partial_{t} P_{U}\left(q_{n}(t)\right) \rightarrow \partial_{t} P_{U}(q(t))$ converges pointwise for all $t \in I$ such that $\|q(t)\|_{L^{2}(\omega)} \neq \gamma$. The pointwise a.e. convergence of $\partial_{t} P_{U}\left(q_{n}(t)\right)$ to $\partial_{t} P_{U}(q(t))$ for $t \in I$ with $\|q(t)\|_{L^{2}(\omega)}=\gamma$ follows from the fact that $\left(q(t), \partial_{t} q(t)\right)=0$ holds for almost all such $t \in I$.

In addition we have the pointwise bound $\left\|\partial_{t} P_{U}\left(q_{n}(t)\right)\right\|_{L^{2}(\omega)} \leq\left\|\partial_{t} q\right\|_{C\left(\bar{I} ; L^{2}(\omega)\right)}$. Hence by Lebesgue dominated convergence theorem, $\partial_{t} P_{U}(q) \rightarrow \partial_{t} P_{U}(q)$ strongly in $L^{2}\left(I ; L^{2}(\omega)\right)$.

Proposition (Proposition 3.4). The projection $P_{U}$ onto $U$ is Bouligand differentiable from $L^{2}(\omega)$ to $L^{2}(\omega)$ with Bouligand derivative given by (3.8).

Proof of Proposition 3.4. Let $q, h \in L^{2}(\omega)$ be given. If $\|q\|_{L^{2}(\omega)} \neq \gamma$, then the claim follows immediately. In the case $\|q\|_{L^{2}(\omega)}=\gamma$ and $(q, h) \geq 0$ it holds $\|q+h\| \geq \gamma$, and the claim is a consequence of the Fréchet differentiability of $q \mapsto \gamma \frac{q}{\|q\|_{L^{2}(\omega)}}$ for $q \neq 0$. Moreover, the convergence order $o\left(\|h\|_{L^{2}(\omega)}\right)$ is uniform with respect to $q$ in bounded subsets of $L^{2}(\omega)$.

It remains to consider the case $\|q\|_{L^{2}(\omega)}=\gamma$ and $(q, h)<0$. If in addition $\|q+h\| \leq \gamma$ holds, then the first-order remainder term is zero. This leaves to discuss the case $\|q+h\|_{L^{2}(\omega)}>\gamma$. Here we find

$$
\gamma^{2}<\|q+h\|_{L^{2}(\omega)}^{2}=\gamma^{2}+2(q, h)+\|h\|_{L^{2}(\omega)}^{2},
$$

which implies the inequality $0<-2(q, h)<\|h\|_{L^{2}(\omega)}^{2}$. The first-order remainder term 
is now given as

$$
\begin{aligned}
& P_{U}(q+h)-P_{U}(q)-P_{U}^{\prime}(q ; h) \\
& =\gamma \frac{q+h}{\|q+h\|_{L^{2}(\omega)}}-q-h \\
& =(q+h)\left(\frac{\gamma}{\|q+h\|_{L^{2}(\omega)}}-1\right) \\
& =\frac{\gamma(q+h)}{\|q+h\|_{L^{2}(\omega)}\left(\gamma+\|q+h\|_{L^{2}(\omega)}\right)}\left(\gamma^{2}-\left(\gamma^{2}+2(q, h)+\|h\|_{L^{2}(\omega)}^{2}\right)\right) \\
& =\frac{\gamma(q+h)}{\|q+h\|_{L^{2}(\omega)}\left(\gamma+\|q+h\|_{L^{2}(\omega)}\right)}\left(-2(q, h)-\|h\|_{L^{2}(\omega)}^{2}\right),
\end{aligned}
$$

where we use $a-b=\frac{a^{2}-b^{2}}{a+b}$. Due to the inequality $0<-2(q, h)<\|h\|_{L^{2}(\omega)}^{2}$, the $L^{2}$-norm of the right-hand side of this expression is of the order $o\left(\|h\|_{L^{2}(\omega)}\right)$ for $h \rightarrow 0$ in $L^{2}(\omega)$, uniformly with respect to $q$ in bounded subsets of $L^{2}(\omega)$.

Proposition (Proposition 3.5). The projection $P_{U_{a d}}$ is Bouligand differentiable from $C\left(\bar{I} ; L^{2}(\omega)\right)$ to $L^{2}\left(I ; L^{2}(\omega)\right)$.

Proof of Proposition 3.5. Let $q \in C\left(\bar{I} ; L^{2}(\omega)\right)$ and a sequence $h_{k} \in C\left(\bar{I} ; L^{2}(\omega)\right)$ be given with $h_{k} \rightarrow 0$ in $C\left(\bar{I} ; L^{2}(\omega)\right)$. Let us define the sequence of first-order remainders

$$
r_{k}:=P_{U_{a d}}\left(q+h_{k}\right)-P_{U_{a d}}(q)-P_{U_{a d}}^{\prime}\left(q ; h_{k}\right) .
$$

We have to show $\left\|r_{k}\right\|_{L^{2}\left(I ; L^{2}(\omega)\right)}=o\left(\left\|h_{k}\right\|_{C\left(\bar{I} ; L^{2}(\omega)\right)}\right)$ as $\left\|h_{k}\right\|_{C\left(\bar{I} ; L^{2}(\omega)\right)} \rightarrow 0$. Due to the result of Proposition 3.4, we have $\left\|r_{k}(t)\right\|_{L^{2}(\omega)}=o\left(\left\|h_{k}(t)\right\|_{L^{2}(\omega)}\right)$ as $k \rightarrow \infty$. Moreover, due to the Lipschitz continuity of projections it holds that $\left\|r_{k}(t)\right\|_{L^{2}(\omega)} \leq$ $2\left\|h_{k}(t)\right\|_{L^{2}(\omega)}$.

By the Lebesgue dominated convergence theorem, we have that the sequence $q_{k}$ defined by $q_{k}(t):=\left\|h_{k}(t)\right\|_{L^{2}(\omega)}^{-1}\left\|r_{k}(t)\right\|_{L^{2}(\omega)}$ converges to zero in $L^{2}(I)$ for all $p \in[1, \infty)$. The claim follows with the inequality

$$
\left\|r_{k}\right\|_{L^{2}\left(I ; L^{2}(\omega)\right)} \leq\left\|h_{k}\right\|_{C\left(\bar{I} ; L^{2}(\omega)\right)}\left(\int_{I}\left\|h_{k}(t)\right\|_{L^{2}(\omega)}^{-2}\left\|r_{k}(t)\right\|_{L^{2}(\omega)}^{2} d t\right)^{1 / 2} .
$$

Proposition (Proposition 4.1). The projection $P_{U}: L^{2}(\omega) \rightarrow L^{2}(\omega)$ is Newton differentiable with Newton derivative given by (4.3).

Proof of Proposition 4.1. We need to argue that

$$
\lim _{\|h\| \rightarrow 0} \frac{1}{\|h\|}\left\|P_{U}(q+h)-P_{U}(q)-D P_{U}(q+h) h\right\|=0
$$

for any $q \in L^{2}(\omega)$; see, e.g., [11, p. 261]. Throughout the proof $\|\cdot\|$ stands for $\|\cdot\|_{L^{2}(\omega)}$.

Let us first consider the mapping $G: L^{2}(\omega) \rightarrow \mathbb{R}$ given by

$$
G(q)=\min \left(1, \frac{\gamma}{\|q\|}\right) .
$$

Then

$$
D G(q)(h)= \begin{cases}0 & \text { if }\|q\| \leq \gamma \\ -\frac{\gamma}{\|q\|^{3}}(q, h)_{L^{2}} & \text { if }\|q\|>\gamma\end{cases}
$$

Copyright ( $\odot$ by SIAM. Unauthorized reproduction of this article is prohibited. 
is a Newton derivative for $G$ at any $q \in L^{2}(\omega)$. Since $G$ is Fréchet differentiable for every $u$ with $\|q\| \neq \gamma$ it suffices to consider the case $\|q\|=\gamma$. Let $\left\{h_{n}\right\}$ be an arbitrary sequence in $L^{2}(\omega)$ converging to 0 . It has subsequences $h_{n}^{1}$ and $h_{n}^{2}$ with $\left\|q+h_{n}^{1}\right\| \leq \gamma$ and $\left\|q+h_{n}^{2}\right\|>\gamma$. For the first we have

$$
\begin{aligned}
& \lim _{n \rightarrow \infty} \frac{1}{\left\|h_{n}^{1}\right\|}\left|G\left(q+h_{n}^{1}\right)-G(q)-D G\left(q+h_{n}^{1}\right) h_{n}^{1}\right| \\
& \quad=\lim _{n \rightarrow \infty} \frac{1}{\left\|h_{n}^{1}\right\|}(1-1-0)=0 .
\end{aligned}
$$

For the second one we find

$$
\begin{aligned}
\lim _{n \rightarrow \infty} \frac{1}{\left\|h_{n}^{2}\right\|}\left|G\left(q+h_{n}^{2}\right)-G(q)-D G\left(q+h_{n}^{2}\right) h_{n}^{2}\right| \\
=\lim _{n \rightarrow \infty} \frac{\gamma}{\left\|h_{n}^{2}\right\|}\left(\frac{1}{\left\|q+h_{n}^{2}\right\|}-\frac{1}{\|q\|}+\frac{1}{\|q\|^{3}}\left(q, h_{n}^{2}\right)\right. \\
\left.\quad \quad-\frac{1}{\|q\|^{3}}\left(q, h_{n}^{2}\right)+\frac{1}{\left\|q+h_{n}^{2}\right\|}\left(q+h_{n}^{2}, h_{n}^{2}\right)\right) \\
\quad \lim _{n \rightarrow \infty} \frac{\gamma}{\left\|h_{n}^{2}\right\|}\left(o\left(\left\|h_{n}^{2}\right\|\right)-\left(\frac{1}{\|q\|^{3}}-\frac{1}{\left\|q+h_{n}^{2}\right\|^{3}}\right)\left(q, h_{n}^{2}\right)+\frac{1}{\left\|q+h_{n}^{2}\right\|^{3}}\left(h_{n}^{2}, h_{n}^{2}\right)\right)=0 .
\end{aligned}
$$

Here we used that for $q \neq 0$

$$
\frac{1}{\|q+h\|}-\frac{1}{\|q\|}+\frac{1}{\|q\|^{3}}(q, h)=o\left(\|h\|^{2}\right) .
$$

In the proof of the following proposition we shall use the fact that $o$ in (7.4) is uniform with respect to $q$ in sets of the form $\{q: \rho \leq\|q\| \leq R\}$, where $0<\rho<R$.

With (7.3) Newton differentiability of $G$ holds. Newton differentiability of $P_{U}$ is now an easy consequence. In fact, we have

$$
\begin{aligned}
P_{U} & (q+h)-P_{U}(q)-D P_{U}(q+h) h \\
= & (q+h) \min \left(1, \frac{\gamma}{\|q+h\|}\right)-q \min \left(1, \frac{\gamma}{\|q+h\|}\right) \\
& +q\left(\min \left(1, \frac{\gamma}{\|q+h\|}\right)-\min \left(1, \frac{\gamma}{\|q\|}\right)\right) \\
& -h \min \left(1, \frac{\gamma}{\|q+h\|}\right)-q D G(q+h) h \\
= & q(G(q+h)-G(q)-D G(q+h) h)=o(\|h\|) .
\end{aligned}
$$

Remark 7.1. Note that in (7.1) we assigned the value 0 to $D G(q)$ for $q$ at the critical value $\|q\|=\gamma$. This corresponds to the equality sign in (4.3) which appears in the first rather than the second line.

Similarly to considerations above, one can prove that the mapping

$$
\tilde{D} G(q)(h)= \begin{cases}0 & \text { if }\|q\|<\gamma, \\ -\frac{\gamma}{\|q\|^{3}}(q, h)_{L^{2}} & \text { if }\|q\| \geq \gamma\end{cases}
$$

is a Newton derivative of $G$ too. Here, the proof has to be modified in the case $\|q\|=\gamma$

Copyright $@$ by SIAM. Unauthorized reproduction of this article is prohibited. 
only for a subsequence $h_{n}^{3}$ with $\left\|q+h_{n}^{3}\right\|=\gamma$. Then one has

$$
0=\left\|q+h_{n}^{3}\right\|^{2}-\|q\|^{2}=\left\|h_{n}^{3}\right\|^{2}+2\left(q, h_{n}^{3}\right)
$$

which implies $\left(q, h_{n}^{3}\right)=-\frac{1}{2}\left\|h_{n}^{3}\right\|^{2}$. Then (7.2) has to be replaced by

$$
\begin{aligned}
& \lim _{n \rightarrow \infty} \frac{1}{\left\|h_{n}^{3}\right\|}\left|G\left(q+h_{n}^{3}\right)-G(q)-D G\left(q+h_{n}^{3}\right) h_{n}^{3}\right| \\
& \quad=\lim _{n \rightarrow \infty} \frac{1}{\left\|h_{n}^{3}\right\|}\left(1-1+\frac{1}{\gamma^{2}}\left(q+h_{n}^{3}, h_{n}^{3}\right)\right)=\frac{1}{2 \gamma^{2}} \lim _{n \rightarrow \infty} \frac{1}{\left\|h_{n}^{3}\right\|}\left(h_{n}^{3}, h_{n}^{3}\right)=0 .
\end{aligned}
$$

Proposition (Proposition 4.2). The projection $P_{U_{a d}}: C\left(\bar{I} ; L^{2}(\omega)\right) \rightarrow L^{2}(I ;$ $\left.L^{2}(\omega)\right)$ is Newton differentiable with Newton derivative given by $\left(D P_{U}\right) q(t)$ as in (4.3).

Proof of Proposition 4.2. Let $q \in C\left(\bar{I} ; L^{2}(\omega)\right)$. Further let $\left\{h_{n}\right\}$ be a sequence in $C\left(I ; L^{2}(\omega)\right)$ with $h_{n} \rightarrow 0$ in $C\left(I ; L^{2}(\omega)\right)$. Let

$$
K_{1}=\{t:\|q(t)\|<\gamma\}, K_{2}=\{t:\|q(t)\|=\gamma\} \text {, and } K_{3}=\{t:\|q(t)\|>\gamma\} \text {. }
$$

We need to estimate

$$
\begin{aligned}
& \lim _{n \rightarrow \infty}\left(\int_{0}^{1}\left\|P_{U}\left(q(t)+h_{n}(t)\right)-P_{U}(q(t))-D P_{U}\left(q(t)+h_{n}(t)\right) h_{n}(t)\right\|^{2} d t\right)^{\frac{1}{2}} \\
& \leq \lim _{n \rightarrow \infty}\left(\left(\int_{K_{1}} z(t) d t\right)^{\frac{1}{2}}+\left(\int_{K_{2}} z(t) d t\right)^{\frac{1}{2}}+\left(\int_{K_{3}} z(t) d t\right)^{\frac{1}{2}}\right)
\end{aligned}
$$

where $z(t)$ denotes the integrand of the integral on the left-hand side. By Lebesgue's bounded convergence theorem

$$
\lim _{n \rightarrow \infty} \int_{K_{1}} z(t) d t=\int_{K_{1}} \lim _{n \rightarrow \infty}\left\|q(t)+h_{n}(t)-q(t)-h_{n}(t)\right\|^{2} d t=0
$$

and hence $\lim _{n \rightarrow \infty} \frac{1}{\left\|h_{n}\right\|_{C\left(I ; L^{2}(\omega)\right)}}\left(\int_{K_{1}} z(t) d t\right)^{\frac{1}{2}}=0$. Similarly by $(7.4)$

$$
\begin{aligned}
& \lim _{n \rightarrow \infty} \frac{1}{\left\|h_{n}\right\|_{C\left(I ; L^{2}(\omega)\right)}}\left(\int_{K_{3}} z(t) d t\right)^{\frac{1}{2}} \\
& \quad=\lim _{n \rightarrow \infty} \frac{1}{\left\|h_{n}\right\|_{C\left(I ; L^{2}(\omega)\right)}}\left(\int_{K_{3}} o\left(\left\|h_{n}(t)\right\|^{4}\right) d t\right)^{\frac{1}{2}}=0 .
\end{aligned}
$$

To obtain the estimate on $K_{2}$ we express $h_{n}$ as $h_{n}(t)=h_{n}^{1}(t)+h_{n}^{2}(t)$, where

$$
h_{n}^{1}(t)=\left\{\begin{array}{l}
h_{n}(t) \text { if }\left\|q(t)+h_{n}(t)\right\| \leq \gamma, \\
0 \text { if }\left\|q(t)+h_{n}(t)\right\|>\gamma,
\end{array} \quad h_{n}^{2}(t)=\left\{\begin{array}{l}
0 \text { if }\left\|q(t)+h_{n}(t)\right\| \leq \gamma \\
h_{n}(t) \text { if }\left\|q(t)+h_{n}(t)\right\|>\gamma
\end{array}\right.\right.
$$

Copyright (c) by SIAM. Unauthorized reproduction of this article is prohibited. 
By (7.5) we find using a splitting similar to (7.3)

$$
\begin{aligned}
& \left(\int_{K_{2}} z(t) d t\right)^{\frac{1}{2}} \\
& =\left(\int_{K_{2}}\left(G\left(q(t)+h_{n}(t)\right)-G(q(t))-D G\left(q(t)+h_{n}(t)\right) h_{n}(t)\right)^{2}\|q(t)\|^{2} d t\right)^{\frac{1}{2}} \\
& =\gamma\left(\int_{K_{2}}\left(G\left(q(t)+h_{n}^{2}(t)\right)-G(q(t))-D G\left(q_{n}(t)+h_{n}^{2}(t)\right) h_{n}^{2}(t)\right)^{2} d t\right)^{\frac{1}{2}} \\
& \leq \gamma^{2}\left(\int_{K_{2}}\left(\frac{1}{\left\|q(t)+h_{n}^{2}(t)\right\|}-\frac{1}{\|q(t)\|}+\frac{1}{\|q(t)\|^{3}}\left(q(t), h_{n}^{2}(t)\right)\right)^{2} d t\right)^{\frac{1}{2}} \\
& \quad+\gamma^{2}\left(\int_{K_{2}}\left(\left(\frac{1}{\|q(t)\|^{3}}-\frac{1}{\left\|q(t)+h_{n}^{2}(t)\right\|^{3}}\right)\left(q(t), h_{n}^{2}(t)\right)\right)^{2} d t\right)^{\frac{1}{2}} \\
& \quad+\gamma^{2}\left(\int_{K_{2}} \frac{1}{\left\|q(t)+h_{n}^{2}(t)\right\|^{6}}\left\|h_{n}^{2}(t)\right\|^{4} d t\right)^{\frac{1}{2}} \\
& \leq \gamma^{2}\left(\int_{K_{2}} o\left(h_{n}^{2}(t)\right)^{2} d t\right)^{\frac{1}{2}} \\
& \quad+\frac{1}{\gamma^{3}}\left(\int_{K_{2}}\left\|h_{n}^{2}(t)\right\|^{4}\left(\|q(t)\|^{2}+\|q(t)\| \cdot\left\|h_{n}^{2}(t)\right\|+\left\|h_{n}^{2}(t)\right\|^{2}\right)^{2} d t\right)^{\frac{1}{2}} \\
& \quad+\frac{1}{\gamma}\left(\int_{K_{2}}\left\|h_{n}^{2}(t)\right\|^{4} d t\right)^{\frac{1}{2}}=o\left(\left\|h_{n}\right\|_{C\left(I ; L^{2}(\omega)\right)}\right),
\end{aligned}
$$

and consequently $\lim _{n \rightarrow \infty} \frac{1}{\left\|h_{n}\right\|_{C\left(I ; L^{2}(\omega)\right)}}\left(\int_{K_{2}} z(t) d t\right)^{\frac{1}{2}}=0$. This concludes the proof.

\section{REFERENCES}

[1] C. Bardos, G. Lebeau, And J. Rauch, Un exemple d'utilisation des notions de propagation pour le contrôle et la stabilisation de problèmes hyperboliques, Rend. Sem. Mat. Univ. Politec. Torino, (1988), pp. 11-31.

[2] R. BuliRsch, Die Mehrzielmethode zur numerischen Lösung von nichtlinearen Randwertproblemen und Aufgaben der optimalen Steuerung, Report of the Carl-Cranz-Gesellschaft, DLR, Oberpfaffenhofen, Germany, 1971.

[3] L. C. Evans, Partial Differential Equations, Grad. Stud. Math. 19, AMS, Providence, RI, 1998.

[4] H. O. FatToRini, The time optimal problem for distributed control of systems described by the wave equation, in Control Theory of Systems Governed by Partial Differential Equations, Academic Press, New York, 1977, pp. 151-175.

[5] H. O. Fattorini, Infinite-dimensional optimization and control theory, Encyclopedia Math. Appl. 62, Cambridge University Press, Cambridge, 1999.

[6] H. O. FATtoRini, Infinite Dimensional Linear Control Systems: The Time Optimal and Norm Optimal Problems, North-Holland Math. Stud. 201, Elsevier Science B.V., Amsterdam, 2005.

[7] R. Griesse, T. Grund, And D. WaChsmuth, Update strategies for perturbed nonsmooth equations, Optim. Methods Softw., 23 (2008), pp. 321-343.

[8] M. Gugat and G. Leugering, $L^{\infty}$-norm minimal control of the wave equation: On the weakness of the bang-bang principle, ESAIM Control Optim. Calc. Var., 14 (2008), pp. 254-283.

[9] M. Gugat, Penalty techniques for state constrained optimal control problems with the wave equation, SIAM J. Control Optim., 48 (2009/10), pp. 3026-3051.

Copyright $@$ by SIAM. Unauthorized reproduction of this article is prohibited. 
[10] A. Haraux, A generalized internal control for the wave equation in a rectangle, J. Math. Anal. Appl., 153 (1990), pp. 190-216.

[11] K. Ito AND K. Kunisch, Lagrange multiplier approach to variational problems and applications, Adv. Des. Control 15, SIAM, Philadelphia, 2008.

[12] K. Ito and K. Kunisch, Semismooth Newton methods for time-optimal control for a class of ODEs, SIAM J. Control Optim., 48 (2010), pp. 3997-4013.

[13] C. Y. Kaya And J. L. NoAKes, Computational method for time-optimal switching control, J. Optim. Theory Appl., 117 (2003), pp. 69-92.

[14] H. B. Keller, Numerical Methods for Two-Point Boundary-Value Problems, Blaisdell, Waltham, MA, 1968.

[15] D. Kinderlehrer and G. Stampacchia, An Introduction to Variational Inequalities and their Applications, Academic Press, New York, 1980.

[16] W. KRABS, On time-minimal distributed control of vibrating systems governed by an abstract wave equation, Appl. Math. Optim., 13 (1985), pp. 137-149.

[17] W. Krabs, On time-minimal distributed control of vibrations, Appl. Math. Optim., 19 (1989), pp. $65-73$.

[18] A. Kröner, K. Kunisch, And B. Vexler, Semismooth Newton methods for optimal control of the wave equation with control constraints, SIAM J. Control Optim., 49 (2011), pp. 830858.

[19] K. Kunisch And D. Wachsmuth, On time optimal control of the wave equation, its regularization and optimality system, ESAIM Control Optim. Calc. Var., to appear.

[20] J.-L. Lions, Control of Distributed Singular Systems, Gauthier-Villars, Paris, 1985

[21] J.-L. Lions, Exact controllability, stabilization and perturbations for distributed systems, SIAM Rev., 30 (1988), pp. 1-68.

[22] E. Meier And A. E. Bryson, Jr., Efficient algorithm for time-optimal control of a two-link manipulator, J. Guidance Control Dynam., 13 (1990), pp. 859-866.

[23] K. D. Phung, G. Wang, And X. Zhang, On the existence of time optimal controls for linear evolution equations, Discrete Contin. Dyn. Syst. Ser. B, 8 (2007), pp. 925-941.

[24] D. Russell, Controllability and stabilizability theory for linear partial differential equations. recent progress and open questions, SIAM Rev., 20 (1987), pp. 189-221.

[25] G. Wang and E. Zuazua, On the equivalence of minimal time and minimal norm controls for internally controlled heat equations, SIAM J. Control Optim., 50 (2012), pp. 2938-2958.

[26] E. ZuAzuA, Controllability of partial differential equations and its semi-discrete approximations, Discrete Contin. Dyn. Syst., 8 (2002), pp. 469-513.

Copyright $@$ by SIAM. Unauthorized reproduction of this article is prohibited. 